\title{
Spectroscopic analysis of the B/Be visual binary HR $1847^{\star, \star \star}$
}

\author{
J. Kubát ${ }^{1}$, S. M. Saad ${ }^{2}$, A. Kawka ${ }^{1}$, M. I. Nouh ${ }^{2}$, L. Iliev ${ }^{3}$, K. Uytterhoeven ${ }^{4}$, D. Korčáková ${ }^{1}$, P. Hadrava ${ }^{5}$, P. Škoda ${ }^{1}$, \\ V. Votruba ${ }^{1,6}$, M. Dovčiak ${ }^{5}$, and M. Šlechta ${ }^{1}$ \\ 1 Astronomický ústav, Akademie věd České republiky, 25165 Ondřejov, Czech Republic \\ e-mail: kubat@sunstel.asu.cas.cz \\ 2 National Research Institute of Astronomy and Geophysics, 11421 Helwan, Cairo, Egypt \\ 3 Institute of Astronomy, Bulgarian Academy of Sciences, 72 Tsarigradsko Shossee Blvd., 1784 Sofia, Bulgaria \\ ${ }^{4}$ Laboratoire AIM, CEA/DSM-CNRS-Université Paris Diderot, CEA, IRFU, SAp, Centre de Saclay, 91191 Gif-sur-Yvette, France \\ 5 Astronomický ústav, Akademie věd České republiky, Boční II 1401, 14131 Praha 4, Czech Republic \\ ${ }^{6}$ Ústav teoretické fyziky a astrofyziky PřF MU, Kotlářská 2, 61137 Brno, Czech Republic
}

Received 24 November 2009 / Accepted 22 May 2010

\section{ABSTRACT}

\begin{abstract}
We studied both components of a slightly overlooked visual binary HR 1847 spectroscopically to determine its basic physical and orbital parameters. Basic stellar parameters were determined by comparing synthetic spectra to the observed echelle spectra, which cover both the optical and near-IR regions. New observations of this system used the Ondřejov and Rozhen 2-m telescopes and their coudé spectrographs. Radial velocities from individual spectra were measured and then analysed with the code FOTEL to determine orbital parameters. The spectroscopic orbit of HR 1847A is presented for the first time. It is a single-lined spectroscopic binary with a B-type primary, a period of 719.79 days, and a highly eccentric orbit with $e=0.7$. We confirmed that HR $1847 \mathrm{~B}$ is a Be star. Its $\mathrm{H} \alpha$ emission significantly decreased from 2003 to 2008. Both components have a spectral type B7-8 and luminosity class IV-V.
\end{abstract}

Key words. binaries: general - stars: emission-line, Be - stars: individual: HR 1847

\section{Introduction}

HR 1847 (HD 36408, BD +16²794, HIP 25950, ADS 4131) is a bright visual binary consisting of two B type stars. Throughout this paper, the component at $\alpha(\mathrm{J} 2000)=053214.14, \delta(\mathrm{J} 2000)=$ +170329.3 will be denoted as HR 1847A (or component A) and the one at $\alpha(\mathrm{J} 2000)=053214.56, \delta(\mathrm{J} 2000)=+170321.8$ as HR 1847B (or component B). The first observations of this binary, which were obtained on 31 December 1782, were reported by William Herschel (see Herschel 1785, star III.93). He observed this binary again on 21 January 1800 (see Herschel 1821, star 124).

Athough this visual binary consists of two bright stars and Plaskett (1919) recommended further observations, it was not studied much during the last century. Since HR 1847 is both an X-ray source (Berghöfer et al. 1996) and an IRAS source (Coté \& Waters 1987), and considering its brightness, lack of observations, and sometimes confusing catalogue information, it has become quite an interesting object for more detailed study.

\section{Summary of known properties of HR 1847}

In this section we summarize the information currently available for both components of HR 1847, which is scattered throughout the astronomical literature, online catalogues, and databases.

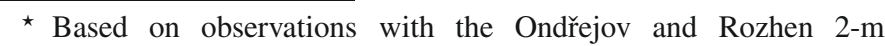
telescopes.

$\star \star$ Appendix is only available in electronic form at http://www . aanda. org

\subsection{Multiplicity}

\subsubsection{Angular separation}

Angular separation $\rho$ and relative position $\theta$ of the components of HR 1847 have already been measured by Friedrich Georg Wilhelm Struve (1837, star No. 730) as an average of 4 measurements obtained in 1829 and $1832\left(\rho=99^{\prime} 81, \theta=141^{\circ} .82\right)$. Later measurements resulted in $\rho=9$. '53, $\theta=140^{\circ} 2$ (Cannon 1912b),

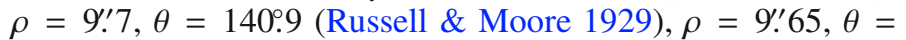
140.85 (Perryman et al. 1997, from HIPPARCOS), $\rho=99^{\prime} 65 \pm$ $0{ }^{\prime} 006, \theta=140.5$ (Fabricius et al. 2002, from the Tycho cata-

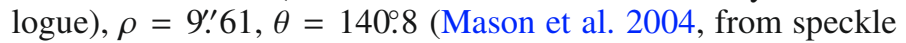
interferometry). The angular separation, as reported by Lindroos (1983) and Abt \& Cardona (1984) is 9.'6.

\subsubsection{Radial velocity variations}

The first radial velocity measurements for both components were reported by Plaskett \& Young (1919) and they showed variability. Plaskett et al. (1920) suggested that both components are spectroscopic binaries. Frost et al. (1926) designated HR 1847A (No. 126 in his Table II) as a spectroscopic binary with an unknown orbit using only one spectrum secured at the Dominion Astrophysical Observatory (DAO) in 1919 by Otto Struve. Component A was also reported to be a spectroscopic binary by Gahm et al. (1983) and Abt et al. (2002). However, no attempt has been made to determine the orbital parameters until now. 
Table 1. HIPPARCOS and UBVRIJHK photometry of HR 1847.

\begin{tabular}{cccc}
\hline \hline band & $\begin{array}{c}\text { HR 1847A } \\
\mathrm{mag}\end{array}$ & $\begin{array}{c}\text { HR 1847B } \\
\mathrm{mag}\end{array}$ & source \\
\hline$H_{p}$ & $6.088 \pm 0.007$ & $6.455 \pm 0.010$ & HIPPARCOS \\
$B_{T}$ & $6.036 \pm 0.008$ & $6.468 \pm 0.004$ & HIPPARCOS \\
$V_{T}$ & $6.057 \pm 0.009$ & $6.458 \pm 0.004$ & HIPPARCOS \\
\hline$U$ & $5.823 \pm 0.030$ & $6.337 \pm 0.030$ & EXPORT \\
$B$ & $6.103 \pm 0.037$ & $6.527 \pm 0.037$ & EXPORT \\
& $6.070 \pm 0.020$ & $6.520 \pm 0.020$ & Maidanak \\
$V$ & $6.090 \pm 0.100$ & $6.490 \pm 0.077$ & EXPORT \\
& $6.090 \pm 0.020$ & $6.510 \pm 0.020$ & Maidanak \\
$R$ & $6.037 \pm 0.100$ & $6.410 \pm 0.077$ & EXPORT \\
& $6.050 \pm 0.020$ & $6.460 \pm 0.020$ & Maidanak \\
$I$ & $6.050 \pm 0.150$ & $6.437 \pm 0.133$ & EXPORT \\
$J$ & $5.964 \pm 0.019$ & $6.280 \pm 0.024$ & 2MASS \\
$H$ & $6.022 \pm 0.021$ & $6.275 \pm 0.021$ & 2MASS \\
$K$ & $6.016 \pm 0.027$ & $6.282 \pm 0.016$ & 2MASS \\
\hline
\end{tabular}

Notes. HIPPARCOS - Perryman et al. (1997), EXPORT - Oudmaijer et al. (2001), Maidanak - Shatskii (1998), 2MASS - Skrutskie et al. (2006).

\subsection{Distance}

The HIPPARCOS parallax of HR 1847 is $2.92 \pm 1.57$ mas (Perryman et al. 1997), which corresponds to a distance of $342 \pm$ $184 \mathrm{pc}$, but the large error reduces the reliability of this distance estimate. However, the new edition of the HIPPARCOS catalogue (van Leeuwen 2007) gives a parallax value of $1.09 \pm$ 2.04 mas, which is unable to provide any information about the distance owing to the very uncertain parallax. Individual parallax measurements of the A and B components are not available.

\subsection{Magnitudes and photometry}

The first reliable magnitude values $\left(V_{A}=6.07, V_{B}=6.44\right)$ were published by Cannon (1912b). She also gives the combined $\mathrm{AB}$ magnitude as 5.49. Maybe that only the combined magnitude value was given in her earlier spectral classification (Cannon 1912a) and in the HD catalogue (Cannon \& Pickering 1918) caused its being incorrectly but often quoted as the magnitude of the A component until now. A similar value is also listed in the Bright Star Catalogue ( $V=5.46$, Hoffleit \& Jaschek 1991).

The photometry of HR 1847 was measured by several authors always during observing runs devoted to large sample of stars. Eggen (1977) measured $u v b y \beta$ photometry of early type stars and derived values $V_{A}=6.1$ and $V_{B}=6.5$. Lindroos (1983) measured $u v b y \beta$ photometry of visual binaries including HR 1847. He derived corresponding $V$ magnitudes of both components from the $y$ filter values as $V_{A}=6.091 \pm 0.019$ and $V_{B}=6.510 \pm 0.023$. $B V R$ observations of visual binaries at Mount Maidanak were performed by Shatskii (1998), and he obtained $V_{A}=6.09 \pm 0.02$ and $V_{B}=6.51 \pm 0.02$. The EXPORT $U B V R I$ photometry (observed by the Nordic Optical Telescope, Oudmaijer et al. 2001) gave values $V_{A}=6.09 \pm 0.10$ and $V_{B}=$ $6.49 \pm 0.07$. Recent determinations of magnitudes were derived from HIPPARCOS photometry (see Perryman et al. 1997). With the HIPPARCOS $V_{T}$ and $B_{T}$ magnitudes, Kharchenko (2001) derived the magnitudes $V_{A}=6.056 \pm 0.008$ and $V_{B}=6.451 \pm$ 0.004 , which do not differ too much from the first values of Cannon. A more complete list of photometric magnitudes of both components of HR 1847 is given in Table 1.

\subsection{Polarization}

Oudmaijer et al. (2001) found the value of $V$ band polarization $P_{V}=0.0063 \pm 0.0004$ for the A component, and $P_{V}=0.0063 \pm$ 0.0002 for the $\mathrm{B}$ component.

\subsection{Spectral types and luminosity classes}

The early classification of both components was "egregie alba" (very white) by Struve (1837). Later, (Cannon 1912b) classified both stars as B9.

Using his own observations in the period from April 1954 to March 1955 at the Yerkes Observatory, Osawa (1959) determined the spectral type of component A as B7IV and of component B as B8IV. From his own observations with the Perkins telescope, Slettebak (1963) determined spectral types B7III for component A and B7IV for component B. Cowley et al. (1969) determined spectral types B8III and B8V for components $\mathrm{A}$ and $\mathrm{B}$, respectively, using spectra from MacDonald and Yerkes Observatories. Levato (1975) observed both components at Cerro Tololo Inter-American Observatory and obtained spectral type B7III for component A and B8IV for B. Finally, Mora et al. (2001) changed the Bright Star Catalogue classification of HR 1847A from B7IIIe to B5V.

\subsubsection{Emission}

Andrews (1968) found that component B shows H $\alpha$ emission using $\mathrm{H} \alpha$ photometry, which was later spectroscopically confirmed by Wackerling (1970). The emission sign, which appeared at the spectral type of component A in both the Bright Star Catalogue and the Simbad database, is incorrect, as $\mathrm{H} \alpha$ emission was never reported for HR 1847A.

\subsection{Rotation}

Rotational velocities of both components were first determined by Slettebak (1963). The projected rotational velocity of component A was measured as $(v \sin i)_{\mathrm{A}}=50 \mathrm{~km} \mathrm{~s}^{-1}$, while component B was found to be rotating rapidly, $(v \sin i)_{\mathrm{B}}=300 \mathrm{~km} \mathrm{~s}^{-1}$. Levato $(1975)$ determined $(v \sin i)_{\mathrm{A}}=60 \mathrm{~km} \mathrm{~s}^{-1}$ and $(v \sin i)_{\mathrm{B}}=$ $300 \mathrm{~km} \mathrm{~s}^{-1}$. Abt et al. (2002) refined the values of the rotational velocities to $(v \sin i)_{\mathrm{A}}=45 \mathrm{~km} \mathrm{~s}^{-1}$ and $(v \sin i)_{\mathrm{B}}=200 \mathrm{~km} \mathrm{~s}^{-1}$ using coudé spectra obtained at the Kitt Peak 0.9-m telescope.

\subsection{Variability}

Slightly different values of magnitude obtained at different times using different instruments, which are listed in the Sect. 2.3, cannot be considered as firm proof of variability. The only available homogeneous set of observations was obtained by the HIPPARCOS satellite. Using HIPPARCOS photometry, Percy et al. (2004) determined the characteristic time scale of variability for HR 1847 as 0.9 days. They conclude that it is a classical Be star, but it is not clear which component they are refering to, since both components A and B have HIPPARCOS photometry available. In addition, data for both components also include several values that correspond to the combined magnitude of both components. Since it is not clear that they excluded the apparently wrong values (those corresponding to combined A+B magnitude), their result is questionable. We performed an independent search for variability and could not confirm the variability time scale found by Percy et al. (2004). 


\subsection{X-rays}

HR 1847 has also been identified as an X-ray source by ROSAT (Berghöfer et al. 1996) with an X-ray luminosity $\log L_{X}=$ 29.64 (in the paper version). In the online version of the same catalogue (their Table 2), this value is slightly different, $\log L_{\mathrm{X}}=29.79$. Both corresponding values of $\log \left(L_{\mathrm{X}} / L_{\mathrm{bol}}\right)=$ -6.82 (or -6.79 in the online version) correspond to a typical $\log \left(L_{\mathrm{X}} / L_{\mathrm{bol}}\right)$ relation for B-type X-ray emitters (cf. Berghöfer et al. 1997). It is, of course, a question of which star of the pair is the X-ray source, if not both. The position of the ROSAT source 1RXS J053214.9+170319 suggests it is more probably coincident with the star TYC 1301-1942-1 = HR 1847B (Flesch \& Hardcastle 2004); however, the positional error is $18^{\prime \prime}$ (Voges et al. 2000), so HR 1847A also lies within the error circle.

\subsection{IR excess}

In their study of IR excess of 101 Be stars Coté \& Waters (1987) found IRAS magnitudes [12] $=4.95$, [25] $=2.22$, $[60]=-0.92$, which places HR 1847 in the [12]-[25]/[12]-[60] colour-colour diagram far away from the region where almost all Be stars are located. Coté \& Waters suggest there is a reflection nebula close to HR 1847. Whitelock et al. (1989) associate HR 1847 with the IRAS SSSC source X0501+589. Magnier et al. (1999) suggest that HR 1847 is a good candidate for a Herbig Ae/Be star with a very large or a very cool circumstellar disk, but Iwata et al. (1999) did not find any CO emission for this star.

Again, it is not clear which star has IR excess, but most likely it is component $\mathrm{B}$, which was found to be a Be star. There is also a small possibility that both stars are Be stars, with component A not in emission at that moment. Further observations may shed a light on it. Coté \& Waters (1987) attributed the spectral type B7IIIe to HR 1847, which corresponds to component B, and $v \sin i=55 \mathrm{~km} \mathrm{~s}^{-1}$, which corresponds to component A.

\subsection{Cluster membership}

Although HR 1847 is located in the same region as the open cluster Collinder 65 (A component is \#771, B component \#772, according to the $\mathrm{WEBDA}^{1}$ database), its membership is improbable mainly because to the different proper motion (Kharchenko et al. 2004).

\section{Observations and data reduction}

The data available for this study consist of several data sets of electronic spectra mostly centred on the $\mathrm{H} \alpha$ region:

\section{Component A}

- 68 spectra in the spectral range 6250-6770 $\AA$ obtained with a CCD SITe ST-005 $800 \times 2000$ pix camera attached to the coudé spectrograph of the $2 \mathrm{~m}$-telescope in Ondřejov (Czech Republic). The spectra were obtained between 18 October 2003 and 25 February 2008. Spectra were reduced using IRAF $^{2}$.

\footnotetext{
1 The Galactic and Magellanic Clouds open cluster database WEBDA is available at http://obswww. unige.ch/webda/

2 IRAF is distributed by the National Optical Astronomy Observatories, which are operated by the Association of Universities for Research in Astronomy, Inc., under cooperative agreement with the National Science Foundation.
}

- One spectrum obtained on 22 March 2003 with the red

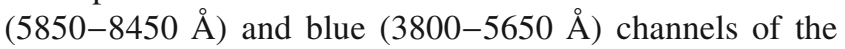
fiber-fed echelle spectrograph HEROS (resolving power $\sim 20000$, for its brief description see Kaufer 1998). The spectrograph was attached to the Cassegrain focus of the 2 m-telescope at the Ondřejov Observatory. All the basic data reduction processing was done using the HEROS pipeline written by Otmar Stahl and Andreas Kaufer as an extension of the basic MIDAS echelle context (see Stahl et al. 1995, also Škoda \& Šlechta 2002).

- 14 spectra obtained in the spectral range (6510-6608 ̊) at Rozhen Observatory (Bulgaria) using the coudéspectrograph of the $2 \mathrm{~m} \mathrm{RCC}$ telescope. The CCD camera Photometrics AT200 with an SITe SI003AB $1024 \times 1024$ CCD chip $(24 \times 24 \mu \mathrm{m}$ pixel size $)$ was used in the $\mathrm{f} / 9.5 \mathrm{cam}-$ era of the spectrograph to provide spectra with a spectral resolution of 36000 in the $\mathrm{H} \alpha$ region. Spectra were obtained between 24 October 2004 and 30 August 2007 and reduced with the MIDAS package.

- One spectrum in the spectral range 6290-6745 ̊̊ obtained at Observatório do Pico dos Dias (OPD, Brasil) and published by Vieiria et al. (2004). For details and information about the reduction process, see the reference above.

In addition to the electronic spectra, we also used the radial velocity measurements of Plaskett \& Young (1919). For comparison purposes, several values of published radial velocities were used from the works of Frost et al. (1926) and Duflot et al. (1992).

Component B spectra came from the same instruments as mentioned in the preceding part describing spectra of component A.

- 38 spectra obtained using the Ondřejov coudé spectrograph between 17 October 2003 and 22 March 2008.

- One HEROS spectrum obtained at the Ondřejov Observatory, one night after the HR 1847A spectrum, on 23 March 2003.

- Three spectra between 28 August 2007 and 30 August 2007 obtained at the Rozhen Observatory.

\subsection{Radial velocity measurements}

Radial velocities were measured using the code SPEFO, which was developed by the late Dr. Jiří Horn (see also Škoda 1996). The FITS files obtained from IRAF and MIDAS were transformed to the SPEFO format, and then the radial velocities (RVs) were obtained interactively by means of the best match of the line profile with its mirror. The RVs were obtained for hydrogen $\mathrm{H} \alpha$, He I $6678 \AA$ A, Si II $6347 \AA$ and $6371 \AA$ lines. RV data for individual components are described later in this paper in Sects. 4.2 and 5.1 .

\section{Component $\mathrm{A}$}

\subsection{The effective temperature and gravity}

The spectral region observed by HEROS covered the range 3800-8620 ̊ (see Figs. A.1 and A.2) and was used to determine the spectroscopic effective temperature and surface gravity. The line spectrum of the star is quite poor. Balmer lines up to H10 and some infrared lines are seen. No emission is present in the Balmer lines and in the lines of other metal ions. 
The effective temperature and surface gravity were determined by comparing the observed spectra to model spectra. We used the ATLAS9 LTE line blanketed model atmospheres, which were calculated by Kurucz (1993) assuming solar metallicity and microturbulent velocity $2 \mathrm{~km} \mathrm{~s}^{-1}$. All synthetic spectra were convolved (using the code ROTIN3 by I. Hubeny) with the Gaussian function having $F W H M=0.25$ to reduce the resolution of the synthetic spectra to the observed resolution. We used a $\chi^{2}$-fitting routine to compare the whole observed spectra with the synthetic ones, and we obtained a best fit of $T_{\text {eff }}=$ $(12500 \pm 500) \mathrm{K}$ and $\log g=3.5 \pm 0.5$. Following the temperature scale of Theodossiou \& Danezis (1991), these values correspond to spectral type B7-B8, and from the value of the surface gravity, we estimate the luminosity class as IV-V. The quoted error bars correspond to the adopted steps of our model grid, which are $500 \mathrm{~K}$ in temperature and 0.5 in $\log g$.

Using the code ROTIN3, we calculated a grid of rotationally broadened spectra for $5 \mathrm{~km} \mathrm{~s}^{-1} \leq v \sin i \leq 500 \mathrm{~km} \mathrm{~s}^{-1}$ with a step of $5 \mathrm{~km} \mathrm{~s}^{-1}$. Then we applied the $\chi^{2}$ fitting routine again to determine the best rotational velocity for this star. To do this, we used the spectral line Mg II $4481 \AA$ (recommended by Gray 1976 , as a line free of pressure broadening) in the fitting process. We determined the projected rotational velocity of HR 1847A as $v \sin i=(40 \pm 3) \mathrm{km} \mathrm{s}^{-1}$, which differs by $5 \mathrm{~km} \mathrm{~s}^{-1}$ from the value of Abt et al. (2002). The error of our value of the rotational velocity was calculated employing a $1-\sigma$ error algorithm of Zhang et al. (1986).

\subsection{Radial velocities}

Tables A.1 and A.2 display the line radial velocity measurements that were derived using the methods described in Sect. 3.1. Columns 1-3 represent the file identification, HJD, and the heliocentric RVs, respectively. Column 4 lists the RV measurements for the $\mathrm{H} \alpha$ line. Columns 5-7 list the RVs measurements for He I $6678 \AA$, Si II $6347 \AA$, and Si II $6371 \AA$ (which are weak) absorption lines, respectively. The measured RVs were shifted to the zero-point using a set of sharp telluric absorption lines by means of the technique described in Horn et al. (1996).

The RVs of HR 1847A vary between $8 \mathrm{~km} \mathrm{~s}^{-1}$ to $27 \mathrm{~km} \mathrm{~s}^{-1}$ with a mean velocity around $13 \mathrm{~km} \mathrm{~s}^{-1}$. Figure 1 displays the measured RVs for hydrogen $\mathrm{H} \alpha$ and He I $6678 \AA$ as a function of time. The plots indicate that our RV measurement started with one value just before the RV maximum, then after the maximum, a clear decrease in the RV values is recorded until the RV values reach their minimum around HJD 2453620 with RV values of $9-10 \mathrm{~km} \mathrm{~s}^{-1}$. Then they quickly reached the next maximum value of $27 \mathrm{~km} \mathrm{~s}^{-1}$ around HJD 2453670 . Although only two cycles are covered by our observations, fortunately we successfully recorded two epochs of rapid rise to the maximum, which happened during a very short time (nearly 35 days) of the long cycle. Rozhen observations confirmed the present distribution of RVs. Such a relatively short event could easily be missed.

The RV measurements that were obtained with different lines in the $\mathrm{H} \alpha$ region show the same distribution with time, and only a small difference in the maximum height can be recognized, as illustrated for the He I $6678 \AA$ line (lower panel of Fig. 1).

\subsection{Period determination}

One of the most fundamental parameters of binary systems is the orbital period. The present RVs span an interval of 1801 days.
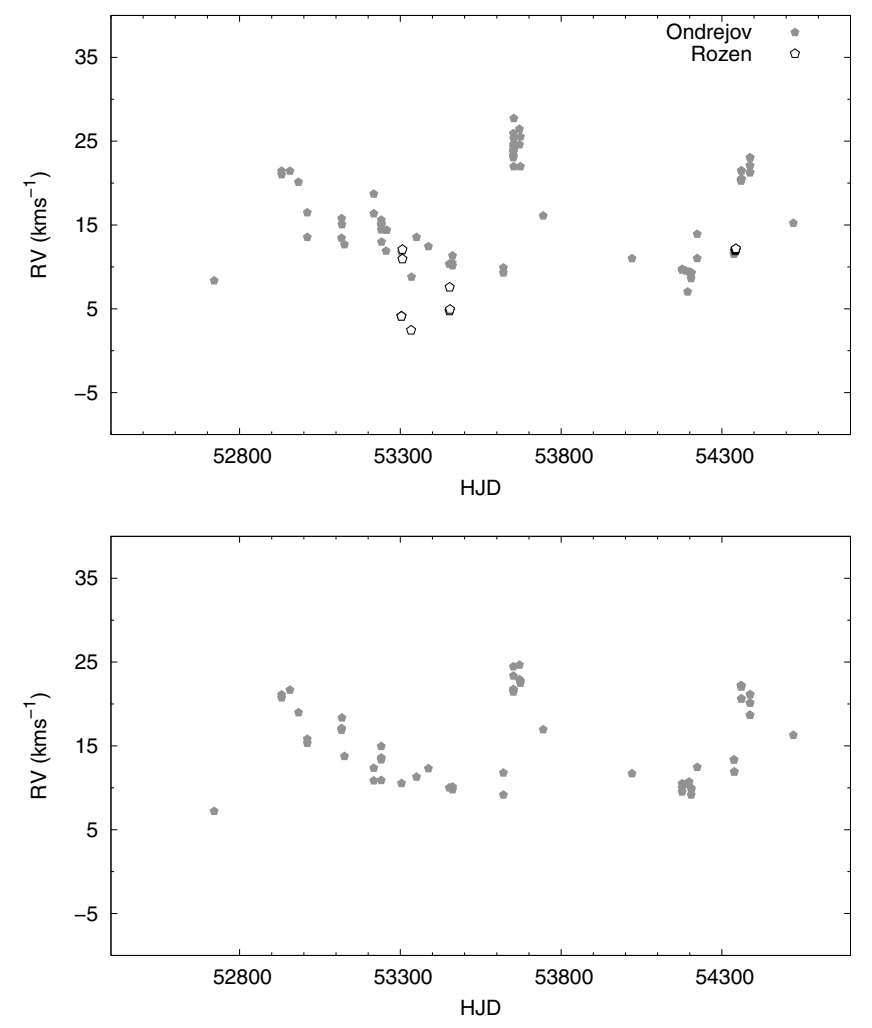

Fig. 1. The radial velocity variations for hydrogen $\mathrm{H} \alpha$ (upper panel) and He I $6678 \AA$ (lower panel) lines measured from the Ondřejov and Rozhen spectrograms as a function of HJD. The He I $6678 \AA$ line is only present in the Ondřejov spectra. Full symbols represent Ondřejov data, and open symbols stand for Rozhen data. All values are from Tables A.1 and A.2.

From the inspection of the time plot one can conclude that

1. the present RV measurements of $\mathrm{H} \alpha$ and of some other metalic lines show clear evidence of long-term cyclic variability; and

2. the relatively large scatter between individual measurements on successive nights indicates that shorter period variability could be present in the data distribution.

No period search of the RV measurements has been carried out so far for HR 1847A. Improper data distribution, gaps, and timing of the observations are the most common problems in the period search process. The period search was carried out separately for several sets of measurements, and two independent numerical period searching routines were used. The first one is based on the phase dispersion minimization (PDM) technique (Stellingwerf 1978), the other one, PERIOD (Breger 1990), is based on a Fourier analysis.

For PERIOD, starting values for the frequencies need to be given, and frequency values are improved within the limits given by the window function. We used this program to search for periodicities in both $\mathrm{H} \alpha$ and $\mathrm{He}$ I $6678 \AA \mathrm{RV}$ data sets in the range between 1 to 1000 days, with an expected frequency resolution of $0.00055 \mathrm{~d}^{-1}$. The upper panel of Fig. 2 displays the periodogram of the detected frequencies in this period range. It shows three candidate frequencies (indicated by arrows in the upper panel of Fig. 2), namely $0.0069 \mathrm{~d}^{-1}, 0.0014 \mathrm{~d}^{-1}$, and $0.1077 \mathrm{~d}^{-1}$. The frequency $0.0069 \mathrm{~d}^{-1}$ has power 61 , and is dominant. The middle panel of Fig. 2 shows the spectral window. In the lower panel of Fig. 2 we show the power spectrum 

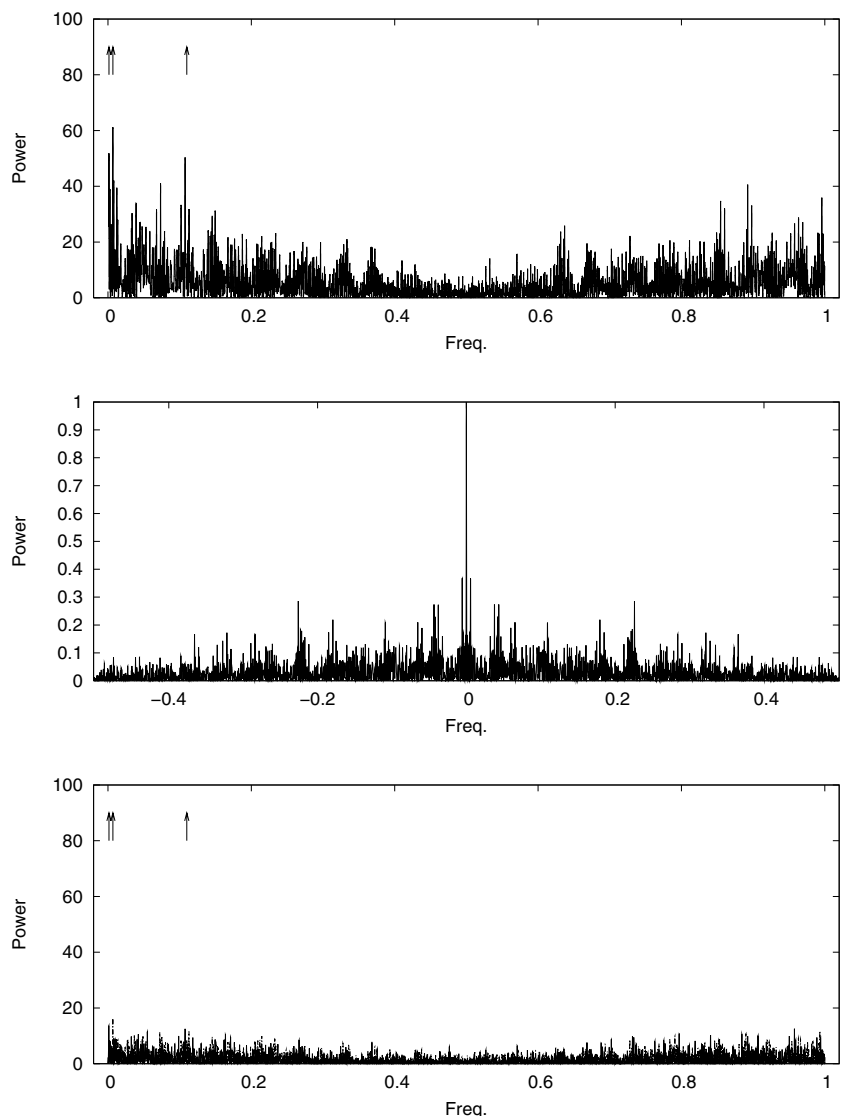

Fig. 2. Upper panel: power spectrum of measured RVs of $\mathrm{H} \alpha$ and He I $6678 \AA$ in the frequency range from $0.001 \mathrm{~d}^{-1}$ to $1 \mathrm{~d}^{-1}$, middle panel: spectral window, and lower panel: power spectrum of the prewhitened residual with $0.0014 \mathrm{~d}^{-1}$. In all panels frequencies at the $x$-axis are given in $\mathrm{d}^{-1}$.

Table 2. Candidate frequencies resulting from the PDM period search.

\begin{tabular}{lrl}
\hline \hline Frequency $\left(\mathrm{d}^{-1}\right)$ & Period (days) & $\theta$ \\
\hline 0.0069 & 144.92753 & 0.3841 \\
0.0014 & 714.28571 & 0.4092 \\
0.1077 & 9.28505 & 0.4668 \\
\hline
\end{tabular}

of the residual periodogram prewhitened for $0.0014 \mathrm{~d}^{-1}$ (using PERIOD), which clearly shows the disappearance of all peaks related to all three candidate frequencies.

Using the PDM technique we searched for periodicity in intervals 1-10 days, 1-100 days, and 1-1000 days. The results are summarized in Table 2 and agree with the ones obtained with PERIOD. Figure 3 illustrates the phase diagrams of measured RVs for the $\mathrm{H} \alpha$ line folded with the $0.1077 \mathrm{~d}^{-1}, 0.0069 \mathrm{~d}^{-1}$ and $0.0014 \mathrm{~d}^{-1}$ frequencies.

Although the frequency $0.0069 \mathrm{~d}^{-1}$ has the highest power (cf. Fig. 2 and Table 2), we suspect that this frequency is not a real one. First, the historical observations of Plaskett \& Young (1919) are out of phase by about half of the period (open squares in the middle panel of Fig. 3). Second, the corresponding RV curve predicts a maximum of RVs near HJD 2453350 , which was not confirmed by our observations. Values around $\sim 15 \mathrm{~km} \mathrm{~s}^{-1}$ appear instead of maximum ones. In addition, the interval of 145 days (or approximately five months) roughly corresponds to the gap between the end of one season in March/April and the next start of observations in
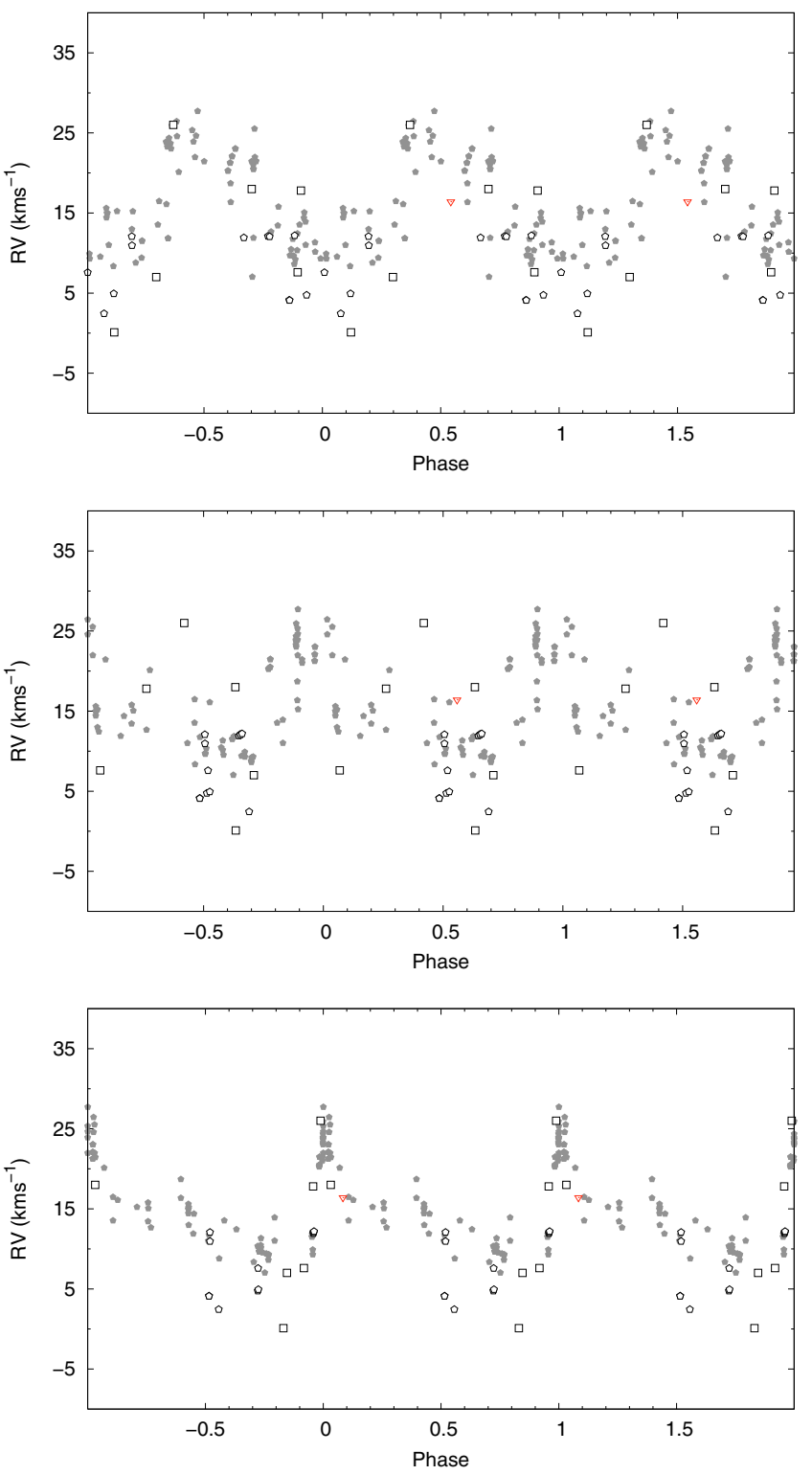

Fig. 3. Phase diagram of measured RVs for $\mathrm{H} \alpha$ lines folded with different frequencies displayed from top to bottom, $0.1077 \mathrm{~d}^{-1}$ (9.28505), $0.00689 \mathrm{~d}^{-1}(144.9275)$, and $0.0014 \mathrm{~d}^{-1}$ (714.2857). Filled circles $(\bullet)$ and open circles (o) denote data obtained from our observations at Ondřejov and Rozhen, respectively. Open squares $(\square)$ and open triangles $(\nabla)$ represent data from Plaskett \& Young (1919) and Vieiria et al. (2004), respectively.

August/September. Third, the candidate frequency $0.0069 \mathrm{~d}^{-1}$ is almost exactly equal to $5 \times 0.0014 \mathrm{~d}^{-1}$. The relative scatter of individual RV values folded with the $0.1077 \mathrm{~d}^{-1}$ frequency is quite large. Following these arguments, we propose the frequency at $0.0014 \mathrm{~d}^{-1}$ as the dominant frequency.

\subsection{Orbital solution}

The variability of the radial velocities with frequency $0.0014 \mathrm{~d}^{-1}$ can most likely be explained by binarity, and it supports the earlier idea of Plaskett \& Young (1919) that the star is a spectroscopic binary.

Starting from the frequency $0.0014 \mathrm{~d}^{-1}$, we performed 4 different orbital solutions using the program FOTEL developed by Hadrava (1990, 2004). For the solutions denoted as I, II, and III 
Table 3. Orbital elements of HR 1847A with FOTEL.

\begin{tabular}{|c|c|c|c|c|}
\hline Element & Solution I & Solution II & Solution III & Solution IV \\
\hline $\mathrm{P}[\mathrm{d}]$ & $715.03 \pm 1.06$ & $713.83 \pm 1.49$ & $719.79 \pm 0.17$ & $710.49 \pm 0.98$ \\
\hline$T_{\text {periast. }}$ & $51501.61 \pm 4.26$ & $51504.34 \pm 5.93$ & $51480.31 \pm 3.88$ & $51503.67 \pm 3.09$ \\
\hline$K\left(\mathrm{~km} \mathrm{~s}^{-1}\right)$ & $7.89 \pm 0.53$ & $7.58 \pm 0.55$ & $7.73 \pm 0.44$ & $8.23 \pm 0.23$ \\
\hline$e$ & $0.79 \pm 0.02$ & $0.72 \pm 0.03$ & $0.70 \pm 0.02$ & $0.73 \pm 0.01$ \\
\hline$\omega[\mathrm{deg}]$ & $306 \pm 5.87$ & $310 \pm 6.99$ & $304 \pm 7.03$ & $286 \pm 3.70$ \\
\hline$\gamma_{1}\left(\mathrm{~km} \mathrm{~s}^{-1}\right)$ & 13.47 & 13.39 & 13.44 & - \\
\hline$\gamma_{2}\left(\mathrm{~km} \mathrm{~s}^{-1}\right)$ & - & 9.54 & 9.88 & - \\
\hline$\gamma_{3}\left(\mathrm{~km} \mathrm{~s}^{-1}\right)$ & - & - & 10.63 & - \\
\hline$\gamma_{4}\left(\mathrm{~km} \mathrm{~s}^{-1}\right)$ & - & - & 10.52 & - \\
\hline$\gamma_{5}\left(\mathrm{~km} \mathrm{~s}^{-1}\right)$ & - & - & - & 13.23 \\
\hline$\gamma_{6}\left(\mathrm{~km} \mathrm{~s}^{-1}\right)$ & - & - & - & 13.36 \\
\hline$\gamma_{7}\left(\mathrm{~km} \mathrm{~s}^{-1}\right)$ & - & - & - & 10.97 \\
\hline$\gamma_{8}\left(\mathrm{~km} \mathrm{~s}^{-1}\right)$ & - & - & - & 13.10 \\
\hline$f(m)$ & 0.0095 & 0.0104 & 0.0126 & 0.0132 \\
\hline No. of RVs & 64 & 76 & 83 & 246 \\
\hline$r m s\left(\mathrm{~km} \mathrm{~s}^{-1}\right)$ & 1.76 & 2.08 & 2.22 & 1.94 \\
\hline
\end{tabular}

Notes. The velocity $\gamma_{1}$ is based on Ondřejov data, $\gamma_{2}$ on Rozhen data, $\gamma_{3}$ on OPD data (Vieiria et al. 2004), and $\gamma_{4}$ on historical data of Plaskett \& Young (1919). In solution IV $\gamma_{5}, \gamma_{6}, \gamma_{7}$, and $\gamma_{8}$ stand for H $\alpha$, He I $6678 \AA$, Si II $6347 \AA$, and Si II $6371 \AA$, respectively.

(see Table 3), the orbital elements were derived for $\mathrm{H} \alpha$ radial velocity variations with data observed from different sites. Solution I is only performed with Ondřejov data, Solution II is obtained using $\mathrm{H} \alpha$ measurements from both Ondřejov and Rozhen observations, Solution III is obtained using all of the available data, namely Ondřejov and Rozhen observations, one spectrum from OPD, and including the historical measurements. Solution IV was obtained using radial velocities of all available spectral lines in the $\mathrm{H} \alpha$ region (H $\alpha$, He I $6678 \AA$, Si II $6347 \AA$, and Si II $6371 \AA$ ).

In our calculations we allowed the period, eccentricity, periastron longitude, and semiamplitude to converge. For each data set FOTEL also allows individual $\gamma$-velocities to be determined. Figure 4 illustrates the FOTEL Solution III with its O-C residuals. We searched for additional periodicities in the residuals but did not find any significant periods.

We adopt the Solution III $(P=719.79 \pm 0.17, e=0.70 \pm$ 0.02 ) as an orbital solution of HR 1847A.

\section{Component B}

The $\mathrm{B}$ component is a Be star with a relatively weak emission in $\mathrm{H} \alpha$ and almost negligible one in $\mathrm{H} \beta$. Since 2003, the weak emission in $\mathrm{H} \alpha$ further weakened, indicating an approaching end of the Be phase. No time scale may be given for this apparently long-term variability, since only 4 years of observations are available. Long term variability (strengthening and weakening of emission) is typical of Be stars on time scales from years to tens of years (for a review see Hubert 2007). For example, $x$ Dra has quite a well-established long term time scale, and its most recent determination is 22.11 years (see Kubát et al. 2010, and references therein). The $\mathrm{H} \alpha$ profiles obtained by our observations are plotted in Fig. 5. We fitted the spectrum of component B using the same methods as for component A. We found $T_{\mathrm{eff}}=(12500 \pm 500) \mathrm{K}, \log g=(3.5 \pm 0.5)$, and $v \sin i=(230 \pm 5) \mathrm{km} \mathrm{s}^{-1}$.
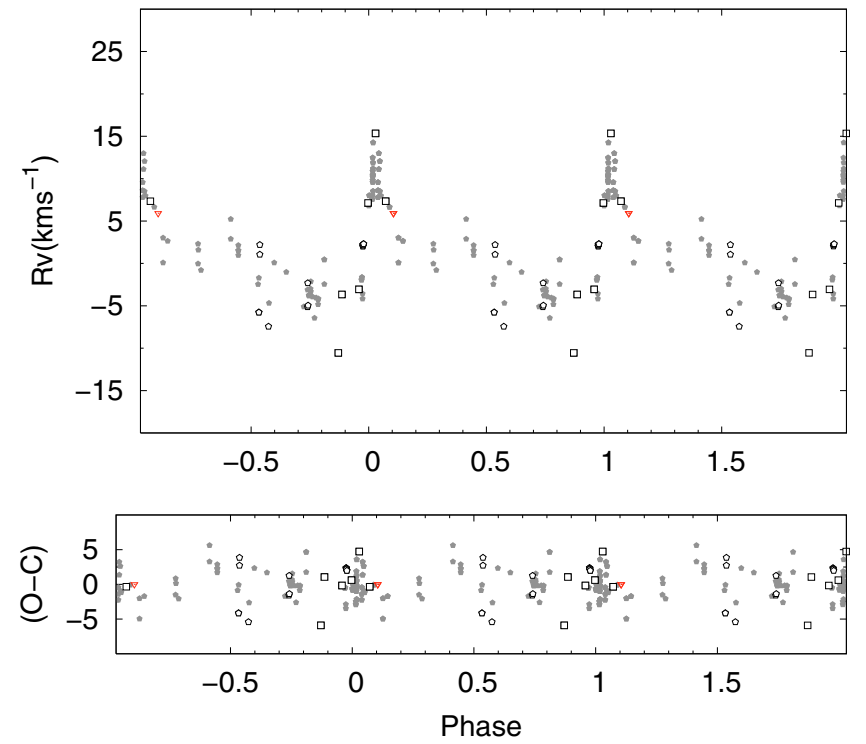

Fig. 4. Upper panel: radial velocity curve of the primary component corresponding to the FOTEL orbital solution III $(P=719$ d.79), which was based on $\mathrm{H} \alpha \mathrm{RV}$ measurements of Ondřejov, Rozhen, and OPD spectra and on RV values from Plaskett \& Young (1919). RV values are displayed in the centre-of-mass reference system. Lower panel: phase plot of $\mathrm{O}-\mathrm{C}$ deviation from the RV curve for Solution III.

\subsection{Radial velocities}

Owing to the high rotational broadening of lines in the component B spectrum, the helium and silicon lines, which were used for RV measurement of component A, are too shallow in component $\mathrm{B}$, and therefore unusable for RV measurement. Consequently, we measured the RVs of component B only from the $\mathrm{H} \alpha$ line. RVs were measured with the profile inversion technique. However, the rapid rotation of the component $\mathrm{B}$ and relatively low $\mathrm{S} / \mathrm{N}$ made it impossibile to arrive at precise $\mathrm{RV}$ values. Cross-correlation techniques, such as least-square deconvolution (LSD, Donati et al. 1997) would not improve the RV accuracy as too few line profiles are available in our spectra. Also, 


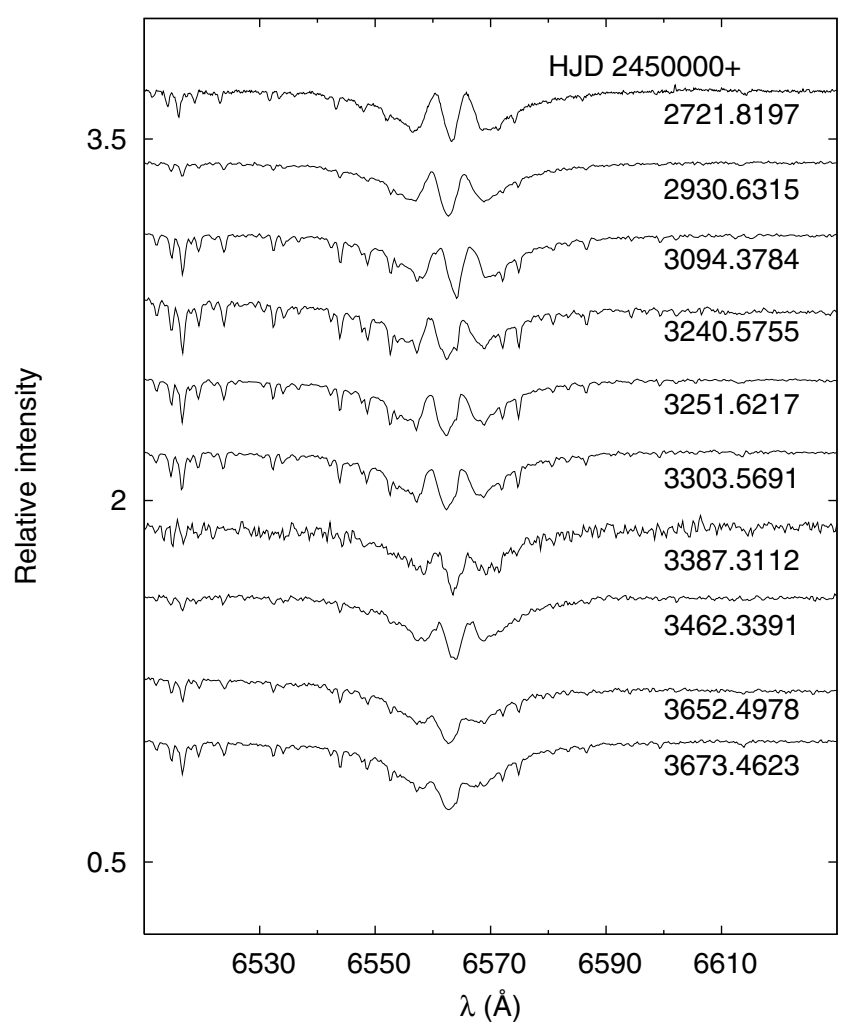

Fig. 5. The evolution of the $\mathrm{H} \alpha$ profile of HR 1847B.

the bisector method does not give more accurate results because of the limited quality and not high enough $\mathrm{S} / \mathrm{N}$ of our spectra.

The $\mathrm{H} \alpha$ line in the B component is characterized by a doublepeak emission with peaks of equal strength. We measured the RVs of three different features at the $\mathrm{H} \alpha$ line, namely of the line wings, of the emission part, and of the central absorption. The results are listed in Tables A.3 and A.4 and plotted in Fig. 6.

Although the RVs are evidently variable, a period search did not give any reasonable result. That is why we cannot make any conclusions about the possible binarity of HR 1847B until a more complete set of more accurate data has been obtained.

\section{The possible $A+B$ system}

There is a question as to whether the components of this visual pair are physically bound. However, there is not enough data to help to resolve this question. The history of all relative position measurements of HR 1847 in the sky (see Sect. 2.1.1) do not allow any conclusion about their relative motion. Slightly different values can be attributed to errors in individual measurements.

Unfortunately, only a common parallax measurement from HIPPARCOS is available. Since individual measurements are missing, we cannot easily say whether the A and B components are at the same distance. However, study of visual binaries by Shatskii (1998) suggests that HR 1847 could be a physical system (with a period shorter than $1 \mathrm{Myr}$ ), since both components have similar proper motion and corresponding photometric parallaxes, which agree with the hypothetical parallax.

In case the visual components of HR 1847 are physically bound, their similar spectral type suggests that they are of similar age. Then there is the question of why one of these stars developed an envelope that causes $\mathrm{H} \alpha$ emission and the other does not. The emission could not be caused by mutual interaction between components $\mathrm{A}$ and $\mathrm{B}$, simply because the distance

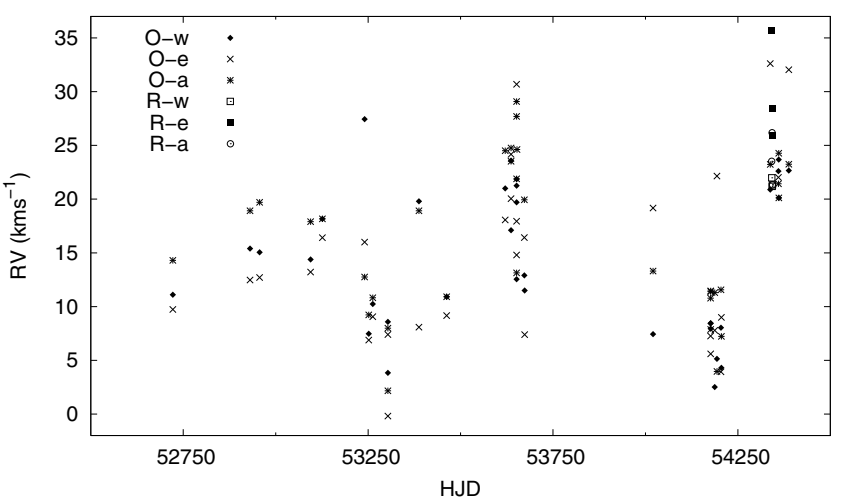

Fig. 6. Time evolution of the $\mathrm{H} \alpha$ radial velocities of HR 1847B. Measurements of line wings are denoted by w, measurements of emission are denoted by e, and measurement of the central absorption are denoted by a. Capital letters denote the observatory, R stands for Rozhen, 0 stands for Ondřejov.

Table 4. Summary of parameters of both components of HR 1847.

\begin{tabular}{ccc}
\hline \hline & HR 1847A & HR 1847B \\
\hline$\alpha(\mathrm{J} 2000)$ & 053214.14 & 053214.56 \\
$\delta(\mathrm{J} 2000)$ & +170329.3 & +170321.8 \\
$V$ & 6.09 & 6.51 \\
\hline$T_{\text {eff }}$ & $12500 \pm 500$ & $12500 \pm 500$ \\
$\log g$ & $3.5 \pm 0.5$ & $3.5 \pm 0.5$ \\
$v \sin i\left(\mathrm{~km} \mathrm{~s}^{-1}\right)$ & $40 \pm 3$ & $230 \pm 5$ \\
Be star & no & yes \\
binary & SB1 & SB $1 ?$ \\
\hline
\end{tabular}

Notes. ${ }^{\star}$ Binary parameters are listed in Table 3.

between the components is too large. A different mechanism for the origin of the Be phenomenon in HR 1847B has to be found (see, e.g., the review by Porter \& Rivinius 2003). The emission in the component B is most probably connected with its rapid rotation. Projected rotational velocity of component A is lower, and this star also has no emission. On the other hand, since the inclination angles are unknown, we can not exclude the possibility that the component $\mathrm{A}$ is also rapidly rotating, as there is apparently no correlation between directions of spin axes for wide binaries (Howe \& Clarke 2009).

\subsection{Comparison with other visual binaries}

HR 1847 is not the only example of a visual multiple system with similar components, with some of them also showing the Be phenomenon. The visual triple system $\beta$ Mon consists of three Be stars (see Marañón di Leo et al. 1994, and references therein), and all these three stars are rapidly rotating (Abt et al. 2002). The projected rotational velocities of $\beta$ Mon $\mathrm{A}$ and $\beta$ Mon $\mathrm{C}$ was reported as $260 \mathrm{~km} \mathrm{~s}^{-1}$ and $250 \mathrm{~km} \mathrm{~s}^{-1}$, respectively, while $\beta$ Mon $\mathrm{B}$ is rotating more slowly $\left(140 \mathrm{~km} \mathrm{~s}^{-1}\right)$ and, interestingly, also has weakest emission (Cowley \& Gugula 1973). This supports the connection between rapid rotation and the Be phenomenon. More visual binaries with Be-components may be found in Abt \& Cardona (1984).

Investigation of such multiple systems with resolved visual components (like HR 1847 or $\beta$ Mon) may help when studying multiple partially resolved or unresolved systems with Be stars like $o$ And (see Olević \& Cvetković 2006, and references therein) or $\beta$ Cep (see Wheelwright et al. 2009, and references 
therein), where light from all stars is mixed, making the analysis extremely difficult. The physical parameters and system characteristics derived from resolved systems will put constraints on stellar structure and binary evolution models, such that the unresolved systems can be modelled in a better way with the improved models. Careful study of resolved systems and careful determination of their parameters are very important feedback on models.

\section{Conclusions}

This paper is the first attempt to collect all available information about the stars in the visual binary system HR 1847 and to correct errors that appear in the SIMBAD database and Bright Star Catalogue. We have presented results of our spectroscopic analysis. HR 1847A ( $V=6.09$, Shatskii 1998) is a B type single-lined, eccentric, $(e=0.70 \pm 0.02)$, spectroscopic binary with a period of $719.79 \pm 0.17$ days. Model atmosphere analysis of this star yielded $T_{\text {eff }}=12500 \mathrm{~K}, \log g=3.5$, and $(v \sin i)_{\mathrm{A}}=40 \mathrm{~km} \mathrm{~s}^{-1}$. HR 1847B $(V=6.51$, Shatskii 1998) is a Be star with variable radial velocities, however, no reasonable orbital solution could be found based on the available data. Model atmosphere analysis of this star yielded the same effective temperature and surface gravity as for the A component, but with a rotational velocity of $(v \sin i)_{\mathrm{B}}=230 \mathrm{~km} \mathrm{~s}^{-1}$. A decrease in the $\mathrm{H} \alpha$ emission was recorded during the last 4.5 years of observations. In addition, positions of X-ray (1RXS J053214.9+170319, see Sect. 2.8) and infrared (X0501+589, see Sect. 2.9) sources indicate that HR $1847 \mathrm{~B}$ is more likely an X-ray and IR source than HR 1847A.

Several other issues, such as the short term variability of HR 1847A or the reliable period determination of HR 1847B, could not be addressed by this paper owing to the lack of necessary data. Consequently, further long-term observations of these stars are desirable. Future work should also include a detailed abundance analysis using high $\mathrm{S} / \mathrm{N}$ spectra and NLTE model atmospheres.

Acknowledgements. The authors would like to devote this paper to the memory of Dr. Izold Pustylnik, with whom they consulted for the history of Struve's observations in Tartu. The authors would also like to thanks Zdeněk Janák, Jan Elner, and Petr Švaříček for their help in early stages of the work. This research made use of the Washington Double Star Catalog maintained at the US Naval Observatory. This research has made use of the NASA's Astrophysics Data System Abstract Service. Our work was supported by a grant of the Grant Agency of the Czech Republic 205/08/0003. The Astronomical Institute Ondřejov is supported by project AV0 Z10030501.

\section{References}

Abbott, D. C. 1980, ApJ, 242, 1183

Abt, H. A., \& Cardona, O. 1984, ApJ, 285, 190

Abt, H. A., Levato, H., \& Grosso, M. 2002, ApJ, 573, 359

Andrews, P. J. 1968, MemRAS, 72, 35

Breger, M. 1990, A\&A, 240, 308

Berghöfer, T. W., Schmitt, J. H. M. M., \& Cassinelli, J. P. 1996, A\&AS, 118, 481 Berghöfer, T. W., Schmitt, J. H. M. M., Danner, R., \& Cassinelli, J. P. 1997, A\&A, 322, 167

Cannon, A. J. 1912a, Ann. Harvard College Observatory, 56, 65

Cannon, A. J. 1912b, Ann. Harvard College Observatory, 56, 227

Cannon, A. J., \& Pickering, E. C. 1918, Ann. Harvard College Observatory, 92, 1

Coté, J., \& Waters, L. B. F. M. 1987, A\&A, 176, 93
Cowley, A., \& Gugula, E. 1973, A\&A, 22, 203

Cowley, A., Cowley, C., Jaschek, M., \& Jaschek, C. 1969, AJ, 74, 375

Donati, J.-F., Semel, M., Carter, B. D., Rees, D. E., \& Collier Cameron, A. 1997, MNRAS, 291, 658

Duflot, M., Fehrenbach, C., Mannone, C., Burnage, R., \& Genty, V. 1992, A\&AS, 110, 177

Eggen, O. J. 1977, PASP, 89, 205

Fabricius, C., Høg, E., Makarov, V. V., et al. 2002, A\&A, 384, 180

Flesch, E., \& Hardcastle, M. J. 2004, A\&A, 427, 387

Frost, E. B., Barrett, S. B., \& Struve, O. 1926, ApJ, 64, 1

Gahm, G. F., Ahlin, P., \& Lindroos, K. P. 1983, A\&AS, 51, 143

Gray, D. F. 1976, The observation and analysis of stellar photospheres (New York: John Wiley \& Sons)

Hadrava, P. 1990, Contrib. Astron. Obs. Skalnaté Pleso, 20, 23

Hadrava, P. 2004, Publ. Astron. Inst. ASCR, 92, 1

Herschel, W. 1785, Phil. Trans. Roy. Soc. London, 75, 40

Herschel, W. 1821, Mem. Astron. Soc., 1, 166

Hoffleit, D., \& Jaschek, C. 1991, The Bright Star Catalogue, 5th Revised Ed. (New Haven: Yale University Observatory)

Horn, J., Kubát, J., Harmanec, P., et al. 1996, A\&A, 309, 521

Howe, K. S., \& Clarke, C. J. 2009, MNRAS, 392, 448

Hubert, A.-M. 2007, in Active OB-Stars: Laboratories for Stellar \& Circumstellar Physics, ed. S. Štefl, S. P. Owocki, \& A. T. Okazaki, ASP Conf. Ser., 361, 27

Iwata, I., Okumura, S., \& Saitō, M. 1999, PASJ, 51, 653

Kaufer, A. 1998, Rev. Mod. Astron., 11, 177

Kharchenko, N. V. 2001, Kin. Fiz. Neb. Tel, 17, 409

Kharchenko, N. V., Piskunov, A. E., Roeser, S., Schilbach, E., \& Scholz, R.-D. 2004, AN, 325, 740

Kubát, J., Saad, S. M., Šlechta, M., \& Yang, S. 2010, in Binaries - Key to Comprehension of the Universe, ed. A. Prša, \& M. Zejda, ASP Conf. Ser., in press

Kurucz, R. L. 1993, ATLAS9 Stellar Atmosphere Programs and $2 \mathrm{~km} \mathrm{~s}^{-1}$ grid, Kurucz CD-ROM No. 13

Levato, H. 1975, A\&A, 19, 91

Lindroos, K. P. 1983, A\&AS, 51, 161

Magnier, E. A., Volp, A. W., Laan, K., van den Ancker, M. E., \& Waters, L. B. F. M. 1999, A\&A, 352, 228

Marañón di Leo, C., Colombo, E., \& Ringuelet, A. E. 1994, A\&A, 286, 160

Mason, B. D., Hartkopf, W. I., Wycoff, G. L., et al. 2004, AJ, 127, 539

Mora, A., Merín, B., Solano, E., et al. 2001, A\&A, 378, 116

Olević, D., \& Cvetković, Z. 2006, AJ, 131, 1721

Osawa, K. 1959, ApJ, 130, 159

Oudmaijer, R. D., Palacios, J., Eiroa, C., et al. 2001, A\&A, 379, 564

Percy, J. R., Harlow, C. D. W., \& Wu, A. P. S. 2004, PASP, 116, 178

Perryman, M. A. C., \& ESA 1997, The Hipparcos and Tycho catalogues, ESA, Noordwijk, ESA SP Ser., 1200

Plaskett, J. S. 1919, JRASC, 13, 197

Plaskett, J. S., \& Young, R. K. 1919, JRASC, 13, 191

Plaskett, J. S., Harper, W. E., Young, R. K., \& Plaskett, H. H. 1920, Publ. Dominion Astrophys. Obs., 1, 163

Porter, J. M., \& Rivinius, Th. 2003, PASP, 115, 1153

Russell, H. N., \& Moore, C. E. 1929, AJ, 39, 165

Shatskii, N. I. 1998, PAZh, 24, 307, Astron. Lett., 24, 257

Škoda, P. 1996, in Astronomical Data Analysis Software and Systems V, ed. G. H. Jacoby, \& J. Barnes, ASP Conf. Ser., 101, 187

Škoda, P., \& Šlechta, M. 2002, Publ. Astron. Inst. ASCR, 90, 40

Skrutskie, M. F., Cutri, R. M., Stiening, R., et al. 2006, AJ, 131, 1163

Slettebak, A. 1963, ApJ, 138, 118

Stahl, O., Kaufer, A., Wolf, B., et al. 1995, J. Astron. Data, 1, 3

Stellingwerf, R. F. 1978, ApJ, 224, 953

Struve, F. G. W. 1837, Stellarum Duplicium et Multiplicium Mensurae Micrometricae, Ex Typographia Academica, Petropoli

Theodossiou, E., \& Danezis, E. 1991, Ap\&SS, 183, 91

van Leeuwen, F. 2007, A\&A, 474, 653

Vieiria, S. L. A., Corradi, W. J. B., Alencar, S. H. P., et al. 2003, AJ, 126, 2971

Voges, W., Aschenbach, B., \& Boller, Th. 2000, IAUC, 7432, 15944

Wackerling, L. R. 1970, PASP, 82, 1357

Wheelwright, H. E., Oudmaijer, R. D., \& Schnerr, R. S. 2009, MNRAS, 497, 487

Whitelock, P. A., Feast, M. W., \& Catchpole, R. M. 1989, MNRAS, 238, 7

Zhang, E.-H., Robinson, E. L., \& Nather, R. E. 1986, ApJ, 305, 740

Pages 9 to 17 are available in the electronic edition of the journal at http: //www . aanda . org 


\section{Appendix A: Visual spectra of HR 1847A and HR 1847B}
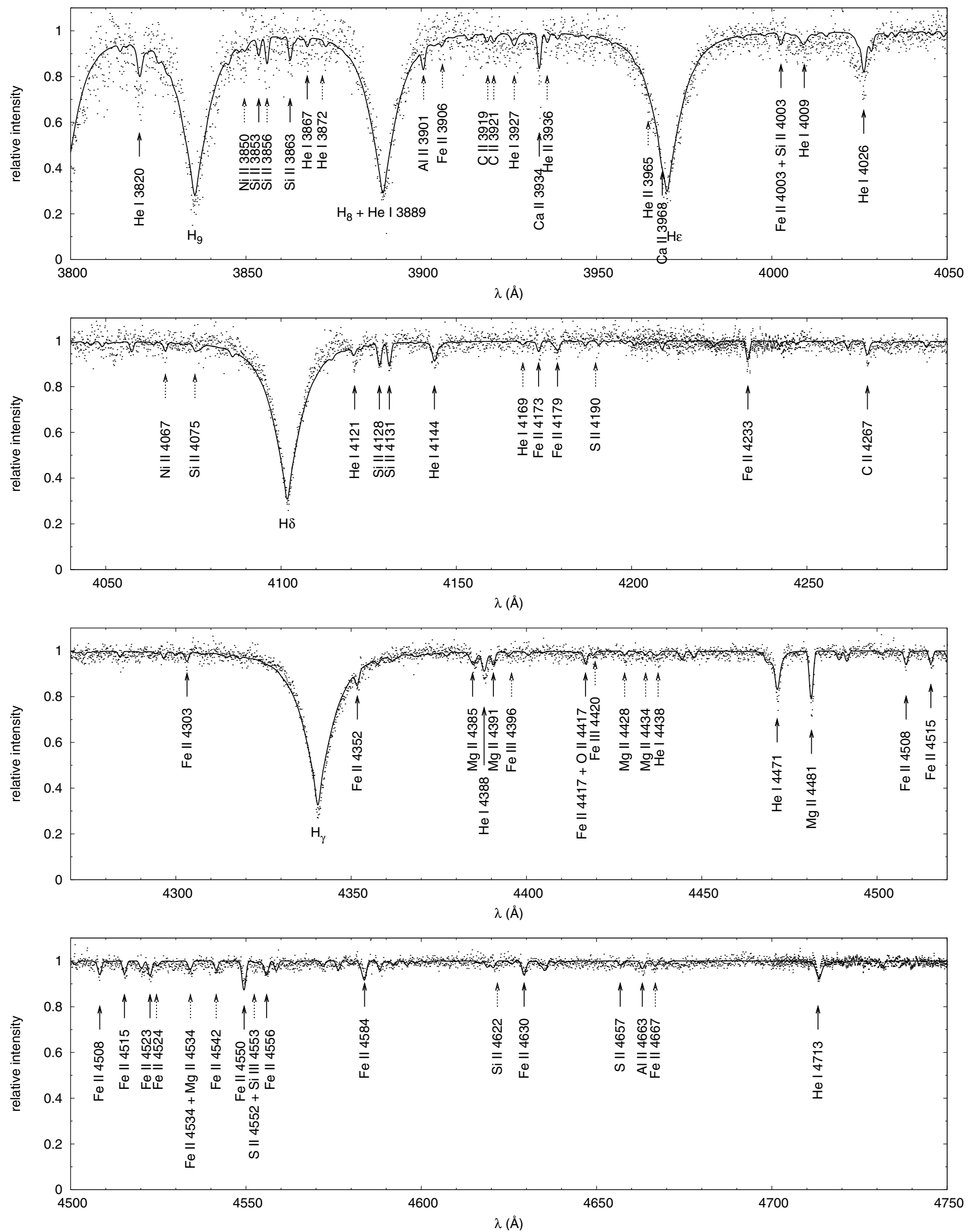

Fig. A.1. Line identification of the spectrum of HR 1847A obtained with the blue channel of the HEROS spectrograph (dots) and comparison with the synthetic spectrum calculated from the Kurucz (1993) LTE model atmosphere $T_{\text {eff }}=12500 \mathrm{~K}, \log g=3.5$, rotationally broadened with $(v \sin i)_{\mathrm{A}}=40 \mathrm{~km} \mathrm{~s}^{-1}$ (full line). 

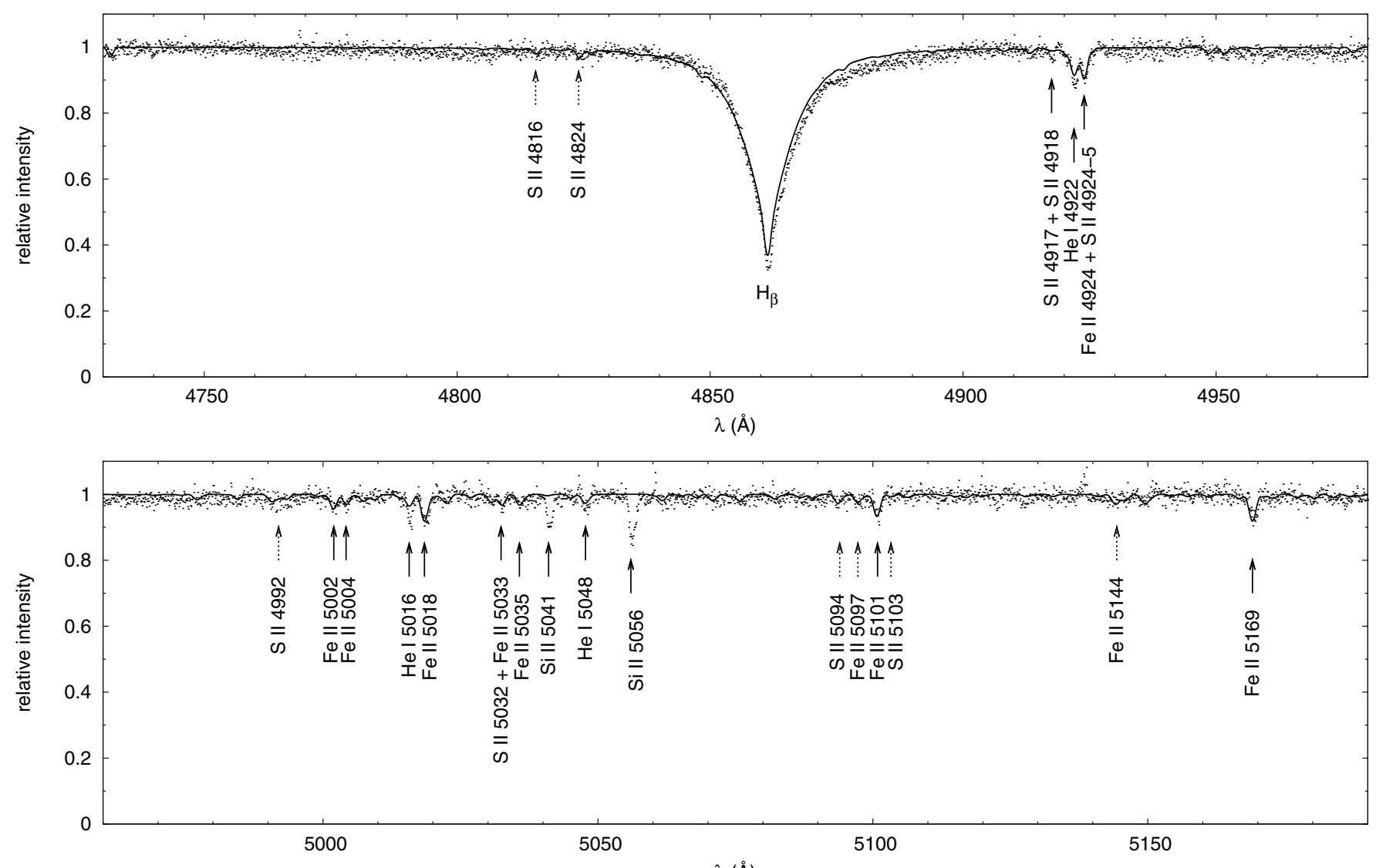

$\lambda(\AA ̊)$
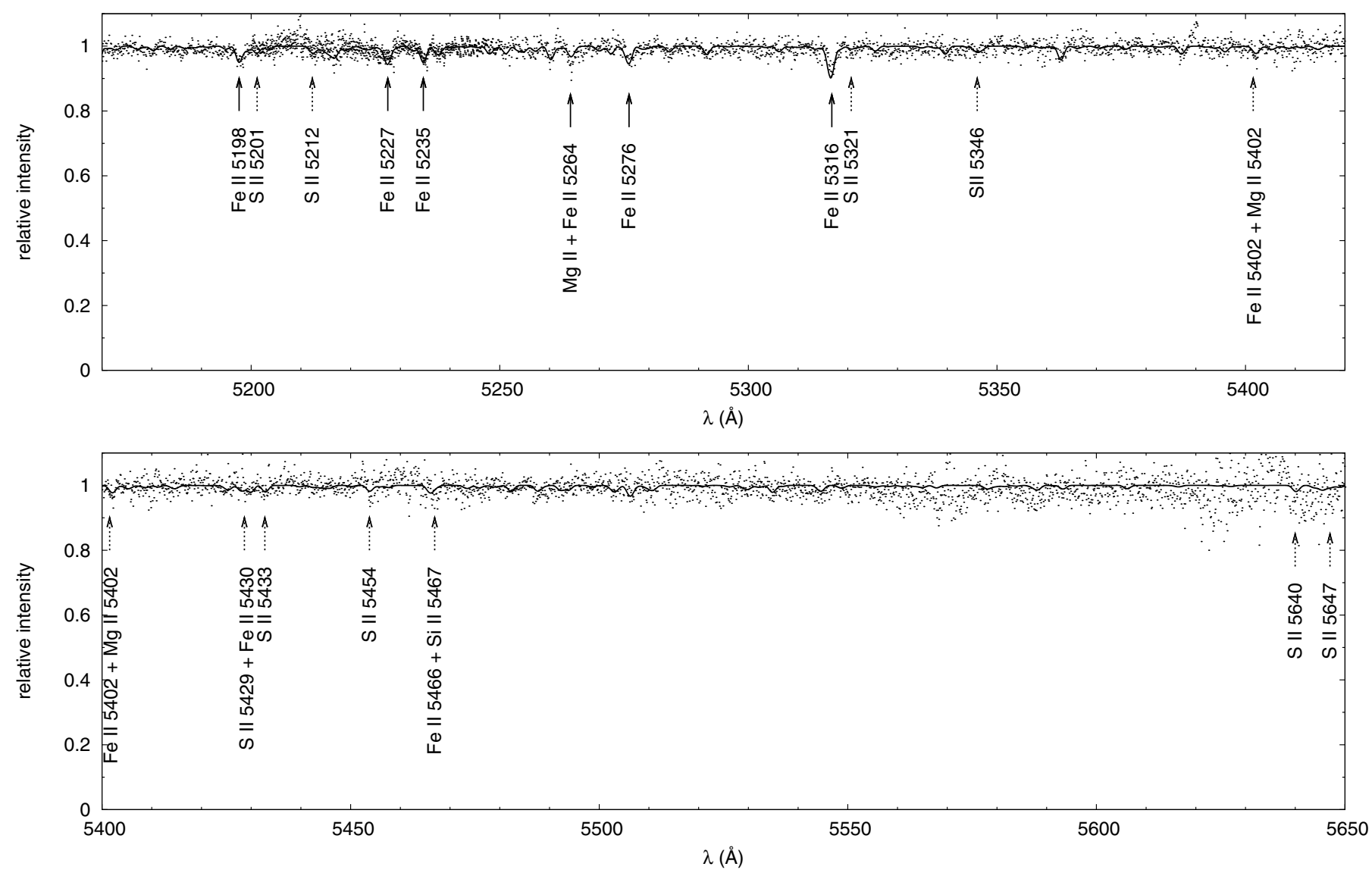

Fig. A.1. continued. 
J. Kubát et al.: Spectroscopic analysis of the B/Be visual binary HR 1847
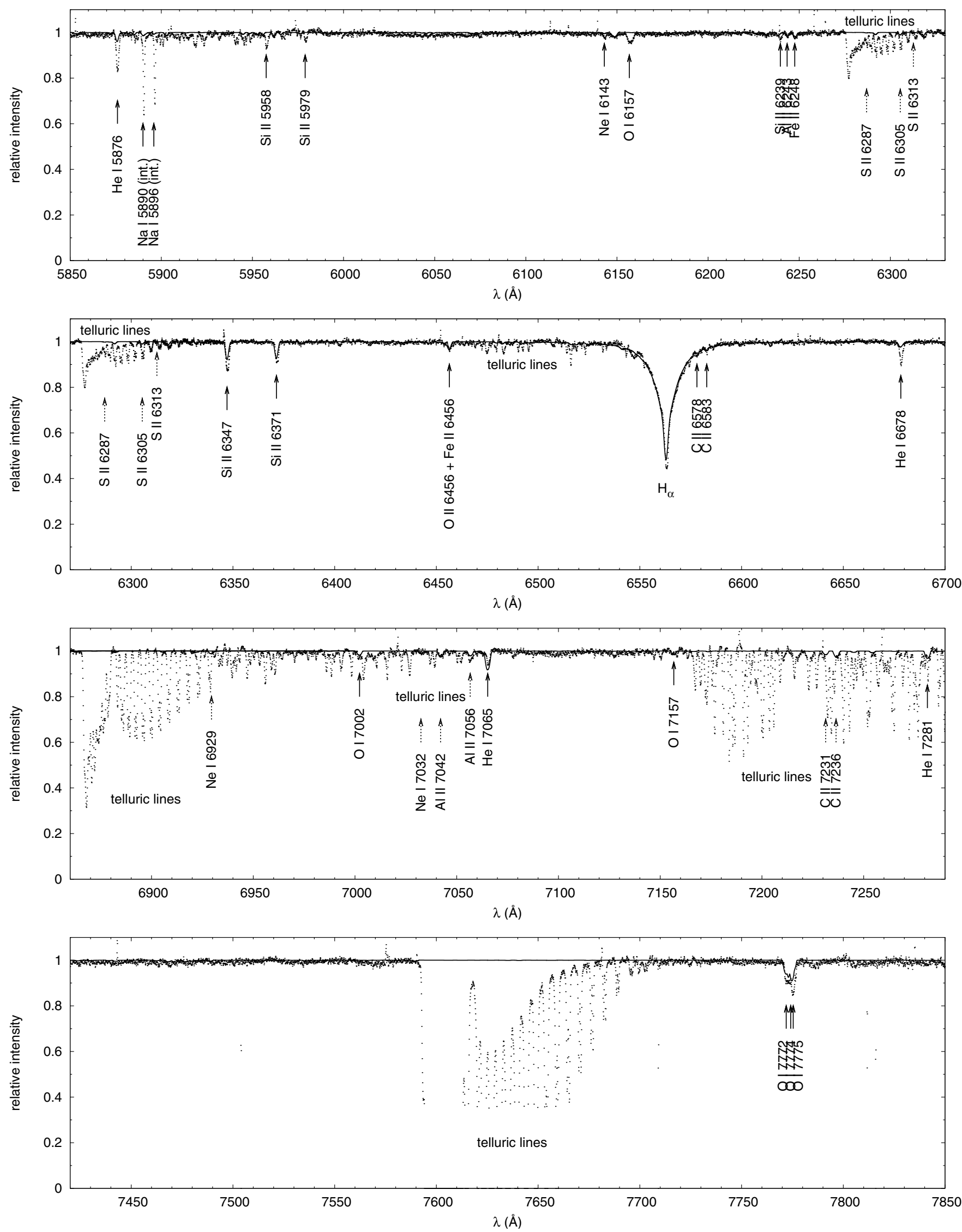

Fig. A.2. Line identification of the spectrum of HR 1847A obtained with the red channel of the HEROS spectrograph (dots) and comparison with the synthetic spectrum calculated from the Kurucz (1993) LTE model atmosphere $T_{\text {eff }}=12500 \mathrm{~K}, \log g=3.5$, rotationally broadened with $(v \sin i)_{\mathrm{A}}=40 \mathrm{~km} \mathrm{~s}^{-1}$ (full line). Note the presence of telluric lines and bands near $6280 \AA$, H $\alpha, 6870 \AA$, $7250 \AA$, and $7590 \AA$. 

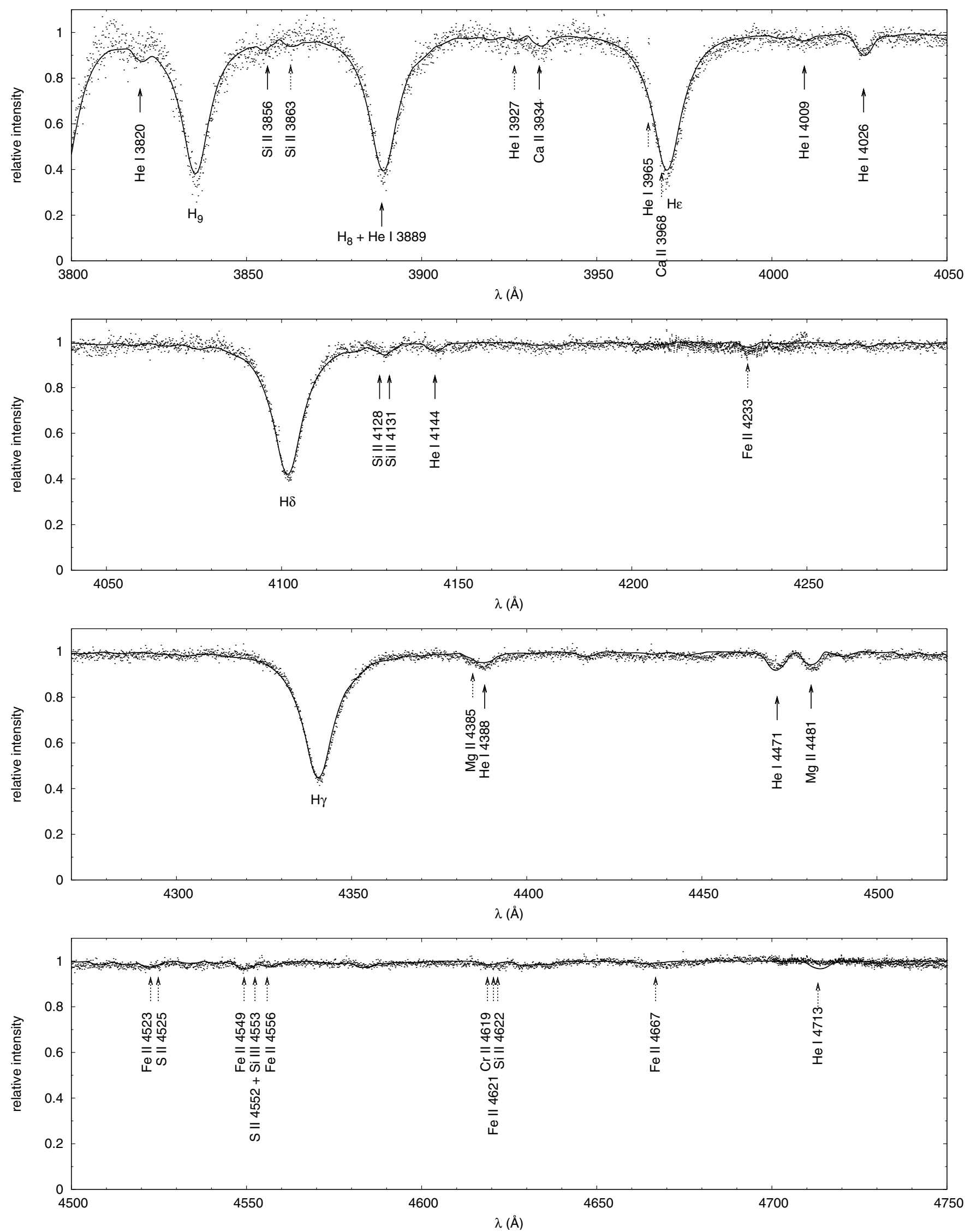

Fig. A.3. Line identification of the spectrum of HR 1847B obtained with the blue channel of the HEROS spectrograph (dots) and comparison with a synthetic spectrum calculated from the Kurucz (1993) LTE model atmosphere $T_{\text {eff }}=12500 \mathrm{~K}, \log g=3.5$, rotationally broadened with $(v \sin i)_{\mathrm{B}}=230 \mathrm{~km} \mathrm{~s}^{-1}$ (full line). 
J. Kubát et al.: Spectroscopic analysis of the B/Be visual binary HR 1847
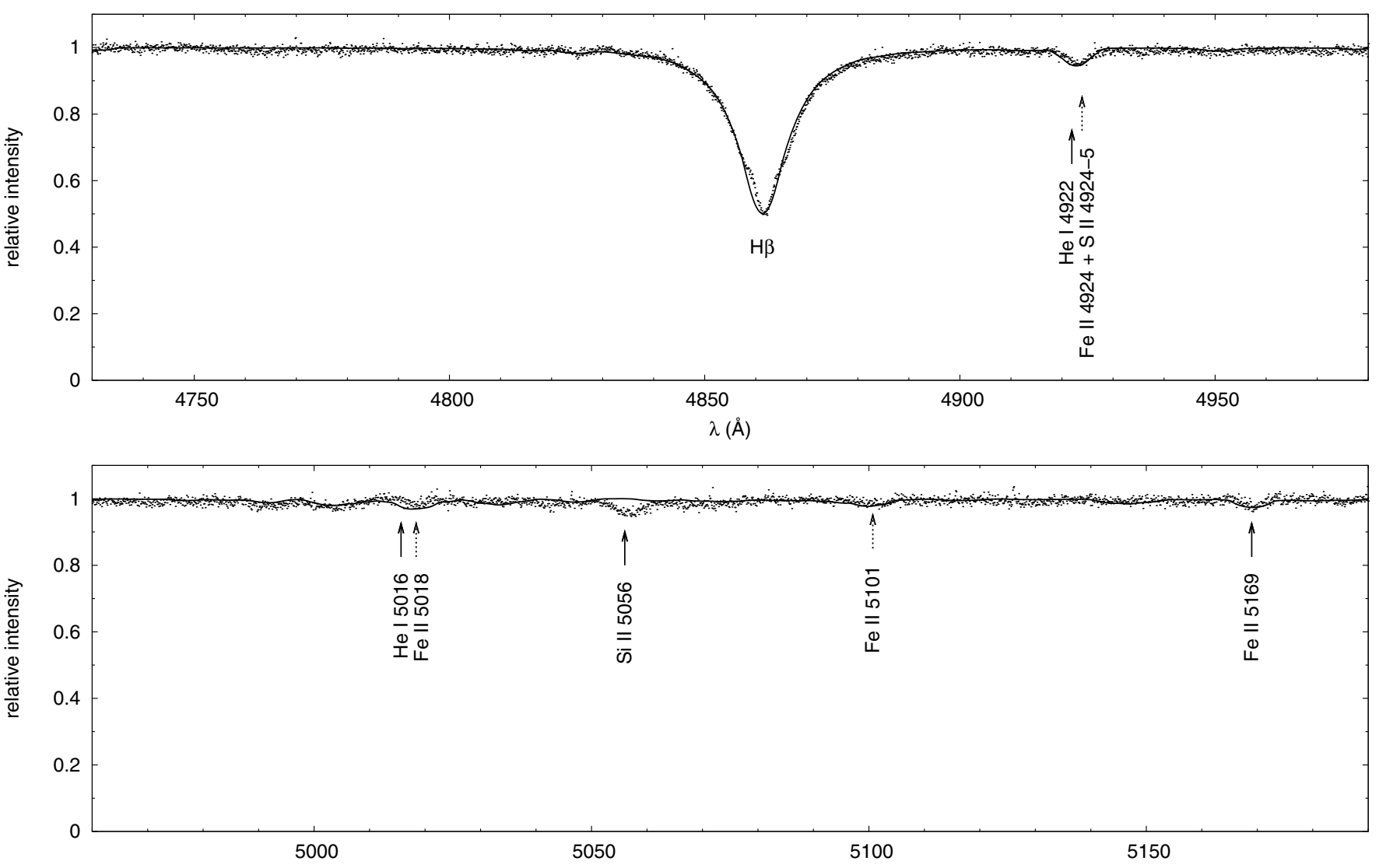

$\lambda(\AA ̊)$
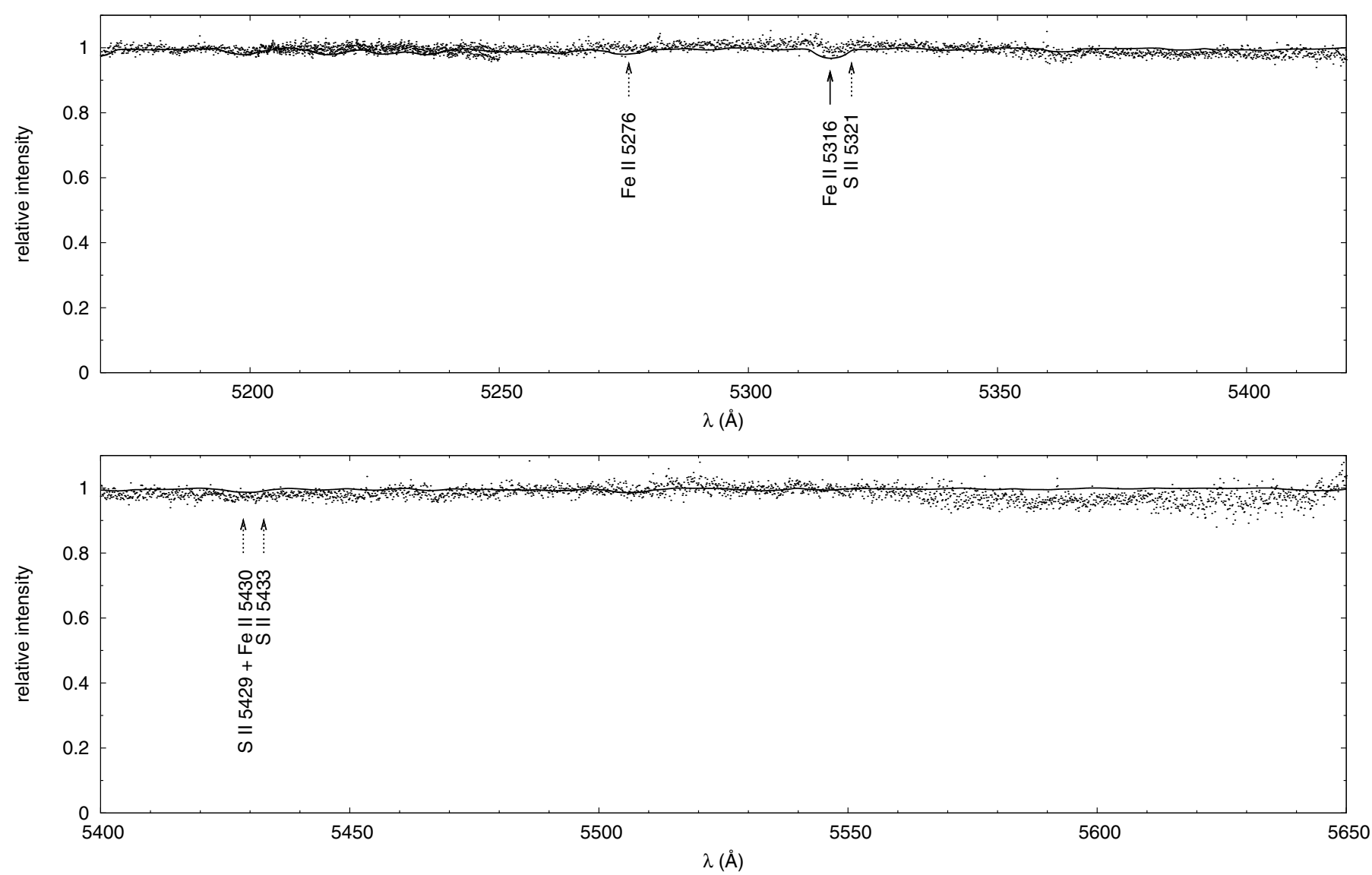

Fig. A.3. continued. 

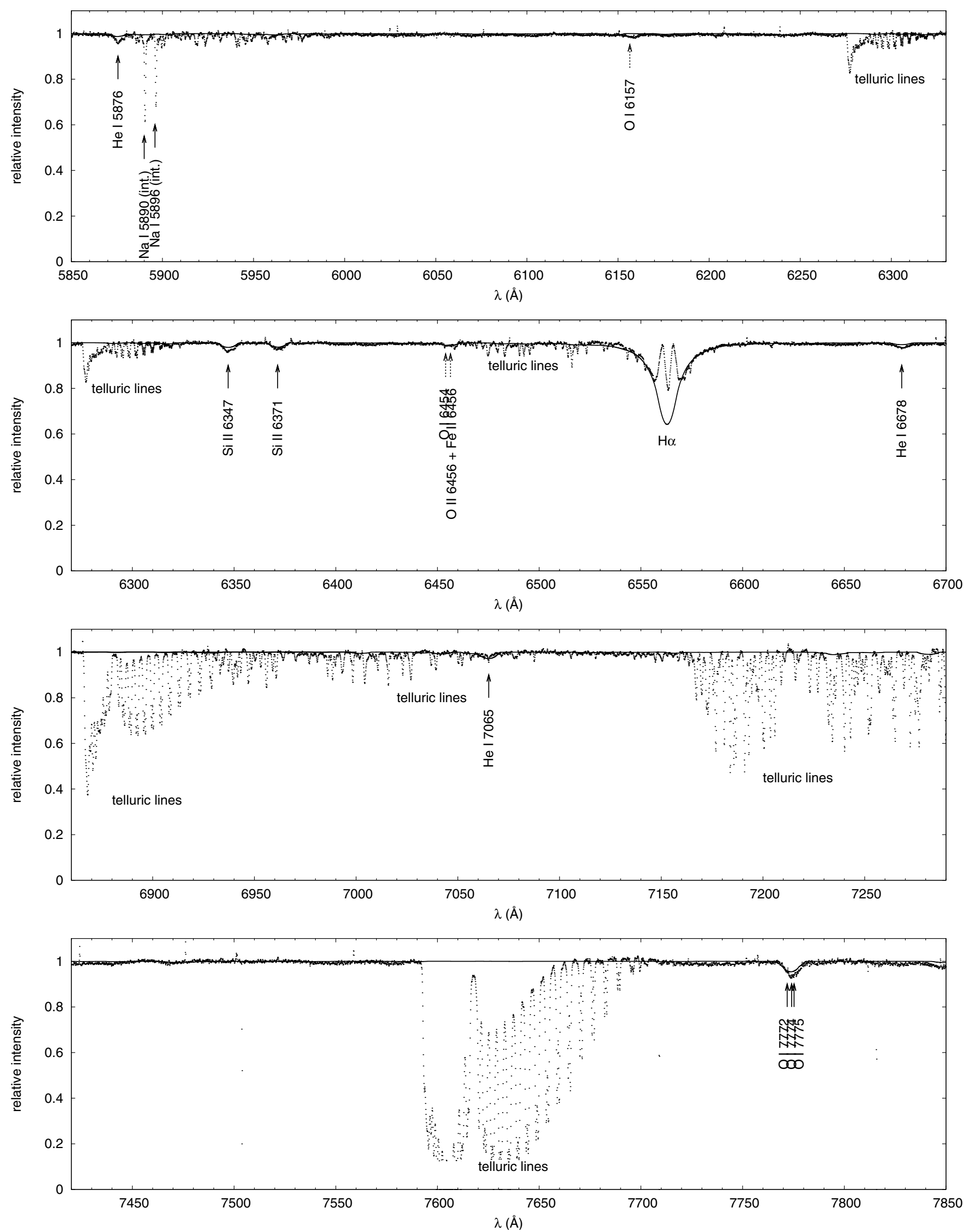

Fig. A.4. Line identification of the spectrum of HR 1847B obtained with the red channel of the HEROS spectrograph (dots), and comparison with a synthetic spectrum calculated from the Kurucz (1993) LTE model atmosphere $T_{\text {eff }}=12500 \mathrm{~K}, \log g=3.5$, rotationally broadened with $(v \sin i)_{\mathrm{B}}=230 \mathrm{~km} \mathrm{~s}^{-1}$ (full line). Note the presence of telluric lines and bands near $6280 \AA, \mathrm{H} \alpha, 6870 \AA, 7250 \AA$, and $7590 \AA$. 
J. Kubát et al.: Spectroscopic analysis of the B/Be visual binary HR 1847

Table A.1. Heliocentric radial velocities (RV) of HR 1847A - spectra from Ondřejov Observatory, where $V_{\text {hel }}$ is the heliocentric velocity.

\begin{tabular}{|c|c|c|c|c|c|c|}
\hline File & $\begin{array}{l}\text { HJD } \\
(J D-2400000)\end{array}$ & $\begin{array}{r}V_{\text {hel }} \\
\left(\mathrm{km} \mathrm{s}^{-1}\right)\end{array}$ & $\begin{array}{l}\mathrm{RV}(\mathrm{H} \alpha) \\
\left(\mathrm{km} \mathrm{s}^{-1}\right)\end{array}$ & $\begin{array}{r}\mathrm{RV}(\mathrm{He} \mathrm{I} 6678 \AA) \\
\left(\mathrm{km} \mathrm{s}^{-1}\right)\end{array}$ & $\begin{array}{r}\mathrm{RV}(\mathrm{Si} \text { II } 6347 \AA) \\
\left(\mathrm{km} \mathrm{s}^{-1}\right)\end{array}$ & $\begin{array}{r}\mathrm{RV}(\mathrm{Si} \mathrm{II} 6371 \AA) \\
\left(\mathrm{km} \mathrm{s}^{-1}\right)\end{array}$ \\
\hline ra5788 & 52721.3728 & -29.71 & & & & \\
\hline$m j 170023$ & 52930.5671 & 25.36 & 21.45 & 21.11 & 18.65 & 20.07 \\
\hline mj 170025 & 52930.6315 & 25.23 & 21.03 & 20.75 & 17.80 & 18.98 \\
\hline $\mathrm{mk} 120038$ & 52956.5608 & 16.02 & 21.45 & 21.67 & 17.67 & 20.76 \\
\hline $\mathrm{ml} 090033$ & 52983.4198 & 3.03 & 20.12 & 18.98 & 16.15 & 18.86 \\
\hline na050019 & 53010.4552 & -11.13 & 13.55 & 15.33 & 14.84 & 16.80 \\
\hline na050021 & 53010.5053 & -11.23 & 16.48 & 15.82 & 13.41 & 16.43 \\
\hline nd210018 & 53117.2973 & -23.56 & 13.44 & 16.89 & 15.73 & 16.70 \\
\hline nd210019 & 53117.3210 & -23.57 & 15.78 & 17.09 & 13.39 & 12.61 \\
\hline nd220014 & 53118.3330 & -23.35 & 15.06 & 18.36 & 13.79 & 16.32 \\
\hline nd300002 & 53126.3175 & -20.47 & 12.67 & 13.76 & 14.90 & 18.87 \\
\hline ng300048 & 53217.5797 & 21.00 & 18.70 & 10.85 & 15.09 & 18.95 \\
\hline ng300049 & 53217.5864 & 21.01 & 16.35 & 12.35 & 12.79 & 17.14 \\
\hline $\operatorname{nh} 220044$ & 53240.5571 & 27.37 & 15.60 & 13.35 & 14.20 & 13.74 \\
\hline $\operatorname{nh} 220045$ & 53240.5649 & 27.36 & 14.43 & 13.58 & 13.35 & 15.67 \\
\hline nh220049 & 53240.5862 & 27.35 & 15.00 & 14.95 & 12.49 & 14.69 \\
\hline nh220053 & 53240.6052 & 26.32 & 14.99 & 10.90 & 13.32 & 15.52 \\
\hline $\operatorname{nh} 230063$ & 53241.5625 & 27.55 & 12.98 & 14.95 & 13.63 & 17.10 \\
\hline ni020057 & 53251.6021 & 28.97 & 15.21 & 15.56 & 11.75 & 15.84 \\
\hline ni060034 & 53255.6179 & 28.14 & 11.90 & 11.14 & 9.90 & 14.66 \\
\hline ni 080043 & 53257.5953 & 29.47 & 14.39 & - & 14.39 & - \\
\hline$n j 240062$ & 53303.5864 & 23.00 & 11.76 & 10.54 & 9.57 & 12.05 \\
\hline nk240049 & 53334.5637 & 10.01 & 8.79 & - & - & - \\
\hline $\mathrm{nl} 100020$ & 53350.2773 & 2.36 & 13.55 & 11.30 & 9.52 & 11.50 \\
\hline oa 160018 & 53387.3315 & -16.40 & 12.44 & 12.31 & 10.18 & 11.89 \\
\hline oc220010 & 53452.3157 & -29.61 & 10.35 & 10.01 & 6.21 & 5.04 \\
\hline oc310022 & 53462.2998 & -28.42 & 11.33 & 9.78 & 6.25 & 11.64 \\
\hline $\operatorname{od} 010016$ & 53461.3659 & -28.62 & 10.47 & 10.03 & 6.91 & 10.42 \\
\hline $\operatorname{od} 010015$ & 53462.2998 & -28.42 & 11.33 & 9.78 & 6.25 & 11.64 \\
\hline $\operatorname{od} 010016$ & 53462.3100 & -28.41 & 10.15 & 10.12 & 5.51 & 10.18 \\
\hline oi060036 & 53620.5833 & 29.34 & 9.90 & 9.15 & 7.15 & 4.46 \\
\hline oi060037 & 53620.5954 & 29.33 & 9.30 & 11.79 & 8.23 & 10.97 \\
\hline oj070018 & 53651.4465 & 27.84 & 23.89 & 21.65 & 20.52 & 20.50 \\
\hline $0 j 070028$ & 53651.4953 & 27.79 & 23.28 & 21.63 & 21.83 & 23.74 \\
\hline oj070034 & 53651.5630 & 27.69 & 24.35 & 21.76 & 20.64 & 22.79 \\
\hline $0 j 070040$ & 53651.6182 & 27.58 & 23.69 & 21.45 & 19.95 & 21.99 \\
\hline oj070042 & 53651.6418 & 27.54 & 23.05 & 23.36 & 20.76 & 21.94 \\
\hline oj070044 & 53651.6703 & 27.48 & 25.93 & 24.46 & 21.79 & 23.70 \\
\hline $0 j 080058$ & 53652.4732 & 27.62 & 25.34 & 26.92 & 25.08 & 25.40 \\
\hline oj080062 & 53652.5220 & 27.56 & 23.91 & 23.42 & 21.44 & 23.02 \\
\hline $0 j 080069$ & 53652.5815 & 27.46 & 21.98 & 23.37 & 20.87 & 22.44 \\
\hline $0 j 080073$ & 53652.6229 & 27.38 & 24.64 & 28.68 & 25.40 & 23.28 \\
\hline oj080079 & 53652.6823 & 27.26 & 27.72 & 25.32 & 23.06 & 27.61 \\
\hline oj260033 & 53670.4313 & 21.60 & 26.45 & 24.66 & 19.67 & 23.76 \\
\hline oj260035 & 53670.4447 & 21.58 & 24.58 & 22.92 & 21.83 & 22.17 \\
\hline oj290036 & 53673.4859 & 20.51 & 25.52 & 22.75 & 20.12 & 21.90 \\
\hline oj290037 & 53673.5018 & 20.48 & 21.98 & 22.49 & 20.33 & 22.87 \\
\hline pa080032 & 53744.3096 & -12.56 & 16.11 & 16.95 & 14.12 & 14.79 \\
\hline pj110024 & 54020.6019 & 26.67 & 11.60 & 11.71 & 9.29 & 12.49 \\
\hline qc160019 & 54176.2664 & -29.87 & 9.03 & 10.00 & 5.16 & 8.53 \\
\hline qc160037 & 54176.3253 & -29.98 & 8.83 & 10.50 & 6.73 & 7.65 \\
\hline qc160046 & 54176.4145 & -30.04 & 9.94 & 9.52 & 6.06 & 8.80 \\
\hline qc 270011 & 54187.3995 & -29.25 & 8.95 & 10.44 & 5.00 & 7.82 \\
\hline $\mathrm{qd} 020010$ & 54193.3620 & -28.37 & 7.03 & 10.44 & 4.54 & 7.95 \\
\hline $\mathrm{qd} 070002$ & 54198.3054 & -27.40 & 9.50 & 10.71 & 6.31 & 8.86 \\
\hline qd130019 & 54204.2939 & -26.03 & 8.63 & 9.98 & 3.03 & 7.47 \\
\hline qd130023 & 54204.3337 & -26.05 & 9.20 & 9.15 & 5.43 & 4.80 \\
\hline qd140022 & 54205.3262 & -25.79 & 9.31 & 9.81 & 5.81 & 8.30 \\
\hline $\mathrm{qe} 020011$ & 54223.3037 & -20.03 & 13.92 & - & - & - \\
\hline qe020012 & 54223.3097 & -20.03 & 11.02 & 12.46 & 2.89 & 6.97 \\
\hline qh240029 & 54337.5997 & 27.59 & 11.53 & 13.34 & 12.39 & 15.14 \\
\hline qh250033 & 54338.6177 & 27.71 & 11.84 & 11.91 & 9.87 & 13.95 \\
\hline qi 150036 & 54359.5365 & 29.70 & 20.28 & 22.21 & 18.66 & 20.35 \\
\hline qi 160031 & 54360.5360 & 29.70 & 20.50 & 22.06 & 19.61 & 23.03 \\
\hline qi 160039 & 54360.6371 & 29.57 & 21.48 & 20.63 & 17.17 & 21.79 \\
\hline$q j 130036$ & 54387.4787 & 26.51 & 21.26 & 18.68 & 21.17 & 20.95 \\
\hline qj 130040 & 54387.5534 & 26.39 & 22.08 & 20.12 & 20.21 & 21.70 \\
\hline qj130046 & 54387.6826 & 26.13 & 23.03 & 21.13 & 19.13 & 21.96 \\
\hline rb250029 & 54522.3202 & -28.60 & 17.53 & 16.29 & 15.33 & 17.04 \\
\hline
\end{tabular}


Table A.2. Heliocentric radial velocities (RV) of HR 1847A - spectra from Rozhen Observatory.

\begin{tabular}{|c|c|c|c|}
\hline File & $\begin{array}{l}\text { HJD } \\
(\mathrm{JD}-2400000)\end{array}$ & $\begin{array}{r}V_{\text {hel }} \\
\left(\mathrm{km} \mathrm{s}^{-1}\right)\end{array}$ & $\begin{array}{r}\mathrm{RV}(\mathrm{H} \alpha) \\
\left(\mathrm{km} \mathrm{s}^{-1}\right)\end{array}$ \\
\hline $04 j 119$ & 53303.4455 & 23.17 & 10.52 \\
\hline $04 j 120$ & 53303.4563 & 23.16 & 9.14 \\
\hline $04 j 303$ & 53306.5530 & 21.99 & 11.16 \\
\hline $04 j 304$ & 53306.5638 & 21.97 & 12.06 \\
\hline $04 \mathrm{k} 109$ & 53333.3218 & 10.99 & 8.39 \\
\hline $05 c 062$ & 53452.6889 & -29.64 & 4.76 \\
\hline $05 c 163$ & 53453.3861 & -29.56 & 7.58 \\
\hline $05 c 235$ & 53454.4102 & -29.47 & 4.93 \\
\hline $07 \mathrm{~h} 050$ & 54341.5910 & 28.25 & 11.92 \\
\hline 87h129 & 54342.5379 & 28.43 & 12.12 \\
\hline O7h135 & 54342.6011 & 28.36 & 12.05 \\
\hline 87h215 & 54343.5844 & 28.52 & 12.19 \\
\hline
\end{tabular}


J. Kubát et al.: Spectroscopic analysis of the B/Be visual binary HR 1847

Table A.3. Heliocentric radial velocities (RV) of HR 1847B - spectra from Ondřejov Observatory.

\begin{tabular}{|c|c|c|c|c|c|}
\hline File & $\begin{array}{l}\text { HJD } \\
(\text { (JD-2 } 400000)\end{array}$ & $\begin{array}{r}V_{\text {hel }} \\
\left(\mathrm{km} \mathrm{s}^{-1}\right)\end{array}$ & $\begin{array}{r}\left(\mathrm{km} \mathrm{s}^{-1}\right) \\
\text { wings }\end{array}$ & $\begin{array}{l}\mathrm{RV}(\mathrm{H} \alpha) \\
\left(\mathrm{km} \mathrm{s}^{-1}\right) \\
\text { emission }\end{array}$ & $\begin{array}{r}\left(\mathrm{km} \mathrm{s}^{-1}\right) \\
\text { absorption }\end{array}$ \\
\hline ra5806 & 52721.8197 & -29.59 & 11.10 & 9.73 & 14.30 \\
\hline $\mathrm{mj} 170025$ & 52930.6315 & 25.23 & 15.40 & 12.47 & 18.92 \\
\hline $\mathrm{mk} 120038$ & 52956.5608 & 16.02 & 15.05 & 12.71 & 19.70 \\
\hline nc290015 & 53094.3765 & -28.89 & 14.39 & 13.21 & 17.90 \\
\hline nd300001 & 53126.3043 & -20.50 & 18.17 & 16.41 & 18.17 \\
\hline nh220047 & 53240.5755 & 27.33 & 27.44 & 16.00 & 12.75 \\
\hline ni020058 & 53251.6217 & 27.36 & 7.47 & 6.88 & 9.22 \\
\hline $\operatorname{ni} 130036$ & 53262.5828 & 29.65 & 10.24 & 9.06 & 10.82 \\
\hline$n j 240060$ & 53303.5565 & 23.02 & 3.83 & -0.19 & 2.16 \\
\hline nj240061 & 53303.5692 & 22.99 & 8.58 & 7.40 & 7.99 \\
\hline oa160016 & 53387.3113 & -16.39 & 19.80 & 8.08 & 18.92 \\
\hline od010017 & 53462.3213 & -28.44 & 10.92 & 9.16 & 10.92 \\
\hline oi060038 & 53620.6195 & 29.30 & 21.00 & 18.06 & 24.51 \\
\hline oi220034 & 53636.6211 & 29.34 & 23.59 & 24.17 & 24.76 \\
\hline oi220035 & 53636.6365 & 29.31 & 17.11 & 20.04 & 23.52 \\
\hline oj070034 & 53651.5630 & 27.69 & 19.70 & 17.94 & 29.08 \\
\hline oj070040 & 53651.6182 & 27.58 & 12.55 & -2.11 & 13.14 \\
\hline oj070042 & 53651.6418 & 27.54 & 21.86 & 30.69 & 21.89 \\
\hline oj070044 & 53651.6703 & 27.48 & 21.25 & 14.80 & 27.70 \\
\hline oj080060 & 53652.4977 & 27.60 & - & 37.52 & 24.62 \\
\hline oj280042 & 53672.5077 & 21.87 & 12.90 & 16.42 & 19.94 \\
\hline oj290035 & 53673.4623 & 21.60 & 11.50 & 7.39 & - \\
\hline pj110026 & 54020.6245 & 26.62 & 7.44 & 19.17 & 13.31 \\
\hline qc160019 & 54176.2664 & -29.87 & 8.43 & 7.26 & 10.78 \\
\hline qc160039 & 54176.3427 & -29.98 & 7.94 & 5.60 & 11.46 \\
\hline qc160048 & 54176.4353 & -30.05 & 8.46 & 7.87 & 11.39 \\
\hline qc2 270010 & 54187.3763 & -29.24 & 2.51 & 7.78 & 11.30 \\
\hline $\mathrm{qd} 020011$ & 54193.3770 & -28.37 & 5.14 & 22.14 & 3.97 \\
\hline qd130021 & 54204.3139 & -26.05 & 8.04 & 3.93 & 11.56 \\
\hline qd140024 & 54205.3461 & -25.80 & 4.30 & 8.99 & 7.23 \\
\hline qh240030 & 54337.6245 & 27.57 & 20.89 & 32.61 & 23.23 \\
\hline qi 150038 & 54359.5627 & 29.68 & 22.60 & 22.02 & 21.43 \\
\hline qi160033 & 54360.5562 & 29.68 & 23.68 & 37.17 & 24.27 \\
\hline qi160039 & 54360.6371 & 29.57 & 20.12 & 37.13 & 20.12 \\
\hline qj130046 & 54387.6826 & 26.13 & 22.66 & 32.04 & 23.25 \\
\hline rb250027 & 54522.2805 & -28.54 & 8.89 & & \\
\hline rb250033 & 54522.3822 & -28.73 & 8.21 & & \\
\hline rb250035 & 54522.4158 & -28.77 & 7.35 & & \\
\hline rc220012 & 54548.3622 & -29.65 & 21.58 & & \\
\hline
\end{tabular}

Table A.4. Heliocentric radial velocities (RV) of HR 1847B - spectra from Rozhen Observatory.

\begin{tabular}{llrrrr}
\hline \hline File & $\begin{array}{l}\text { HJD } \\
(\mathrm{JD}-2400000)\end{array}$ & $\begin{array}{r}V_{\text {hel }} \\
\left(\mathrm{km} \mathrm{s}^{-1}\right)\end{array}$ & $\begin{array}{r}\mathrm{RV}(\mathrm{H} \alpha) \\
\left(\mathrm{km} \mathrm{s}^{-1}\right) \\
\text { wings }\end{array}$ & $\begin{array}{r}\left(\mathrm{km} \mathrm{s}^{-1}\right) \\
\text { emission }\end{array}$ & $\begin{array}{r}\left(\mathrm{km} \mathrm{s}^{-1}\right) \\
\text { absorption }\end{array}$ \\
\hline 07h049 & 54341.5732 & 28.25 & 21.98 & 35.69 & 23.52 \\
07h136 & 54342.6176 & 28.33 & 21.19 & 28.47 & 26.19 \\
07h216 & 54343.5993 & 28.50 & 21.33 & 25.90 & 21.33 \\
\hline
\end{tabular}

